\title{
Mathematical Modelling of the Spread and Control of Onchocerciasis in Tropical Countries: Case Study in Nigeria
}

\author{
Ikechukwu Chiwueze Oguoma and Thomas Mbah Acho \\ Department of Mathematics and Applied Mathematics, Faculty of Natural and Agricultural Sciences, University of the Free State, \\ Bloemfontein 9300, South Africa
}

Correspondence should be addressed to Ikechukwu Chiwueze Oguoma; ikeoguoma@yahoo.com

Received 2 May 2014; Accepted 2 June 2014; Published 21 October 2014

Academic Editor: Abdon Atangana

Copyright (C) 2014 I. C. Oguoma and T. M. Acho. This is an open access article distributed under the Creative Commons Attribution License, which permits unrestricted use, distribution, and reproduction in any medium, provided the original work is properly cited.

Onchocerciasis, also known as river blindness and Robles disease, is a parasitic disease caused by Onchocerca volvulus, a nematode (roundworm), and it is endemic in tropical countries like Nigeria. The objective of this paper is to discuss the mathematical formulation underpinning the spread and control of this disease on one hand. On the other hand, we make use of some new analytical methods to derive the solution of the resulting set of equations. The numerical results are presented to test the efficiency and the accuracy of both methods. The techniques used for solving these problems are friendly, very easy, and less time consuming. The numerical solutions in both cases display the biological behaviour of the real life situation.

\section{Introduction}

Onchocerciasis is a country-wide public health problem in Nigeria. The present estimates had suggested that 7-10 million Nigerians are infected with Onchocerca volvulus, and approximately 40 million are at risk of the disease [1]. More than 120,000 cases of Onchocerciasis related blindness have been reported [2-4], and thousands of people suffer from disabling complications of this disease [5]. Onchocerciasis, which is the world's second-leading infectious cause of blindness, is a parasitic disease caused by the filarial nematode, Onchocerca volvulus. It is termed as "river blindness" because it is spread by the bite of the blackfly vector that breads near oxygen-high fast-flowing streams and rivers people rely on for washing, drinking, and farming which results in depopulation of the fertile river valleys. The lifespan of blackflies is short, lasting only 2-3 weeks. The disease affects rural communities in Nigeria and is the major cause of blindness and skin disease in endemic areas with serious socioeconomic effects. It has been identified by the World Health Organization as one of the neglected tropical diseases (NTD) $[6,7]$, "Neglected" because they are not mentioned in the millennium Development Goals. This implied that they are not usually included among the important development discussions, and as such they do not receive adequate attention or funding.

Figure 1 explains the life cycle of the parasite Onchocerca volvulus.

Onchocerca volvulus is transmitted to humans through the bite of a blackfly which introduces immature larva forms of the parasite (infective larvae) into their human host.

The larvae migrate to the subcutaneous tissue where they undergo further development and form skin nodules as they mature into adult worms (macrofilaria). The adult worms mate and produce millions of microscopic larvae (microfilaria). This continues for years of the adult worm's life. Although the infection with larvae begins immediately, the disease may not become apparent in an individual for months to years. In most individuals, it develops slowly in the skin, while some patients may show symptoms initially with eye problems, ocular involvement, and general debilitation.

The blackflies absorb the microfilariae as they feed during the day, which further undergo developmental stages within the blackflies into infective larvae, which are then transmitted to the next human victim.

A study was carried out in Imo River Basin, Imo State, Southeastern Nigeria, to assess the prevalence and intensity of 


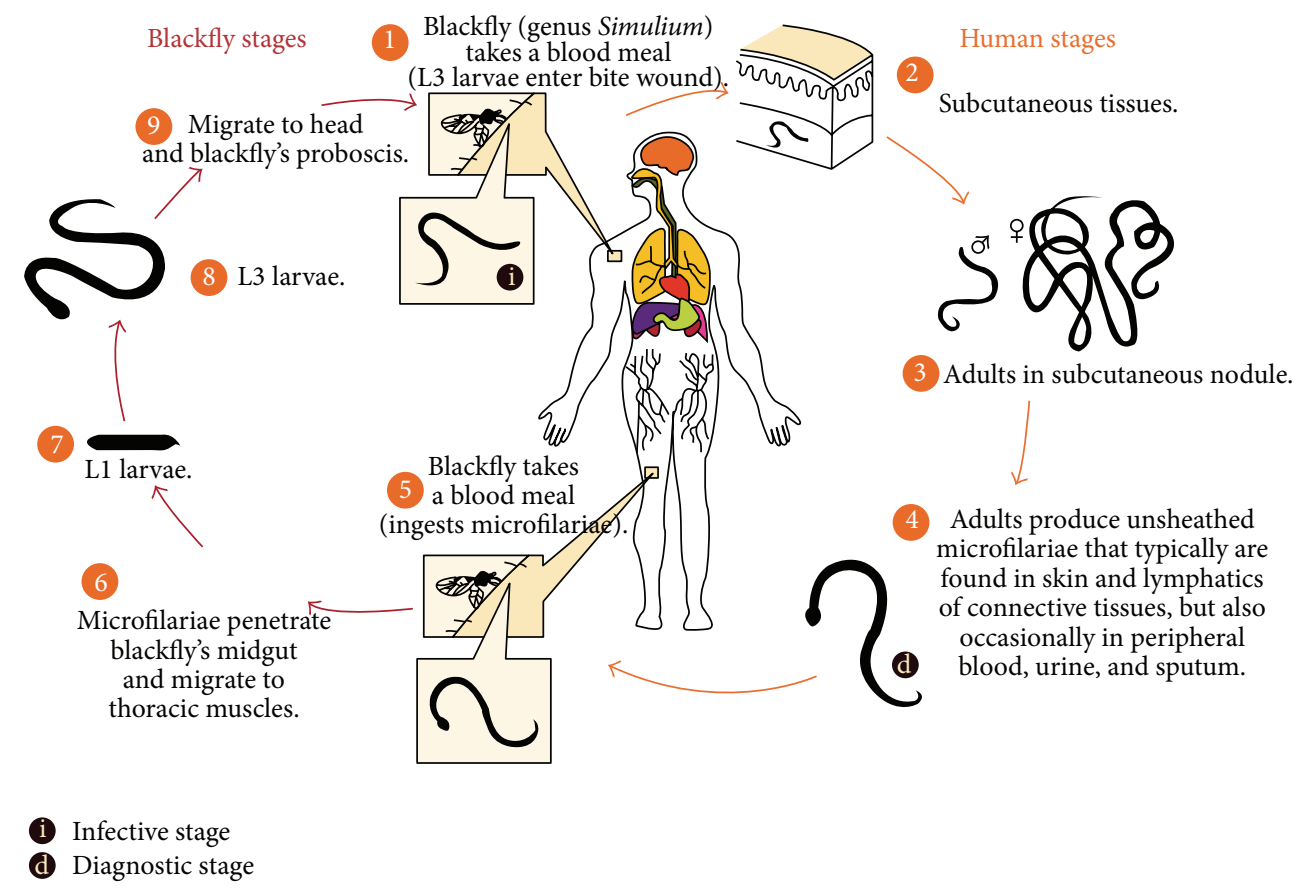

FIgURE 1: Life cycle of Onchocerca volvulus [8].

microfilaria of Onchocerca volvulus in the area [9]. The survey coverage was about $91.8 \%$ of the study population and it was found by EC. Uttah found that thirty-seven percent of those examined was positive for Onchocerca volvulus microfilariae (39.2\% of males and $34.9 \%$ of females). The microfilariae (mf) prevalence increased steadily with increasing age to reach $70.4 \%$ in the oldest age group.

Going towards the northern part of Nigeria, the endemicity of human onchocerciasis was assessed by Anosike and Onwuliri [10] in eight rural communities at risk, in Ningi Local Government Area, Bauchi State, Nigeria, between July 1990 and March 1991. Of the 1536 subjects skin-snipped, 334 (21.7\%) were positive for Onchocerca volvulus microfilariae.

Wogu and Okaka investigated the overall prevalence of onchocerciasis in Okpuje, Owan West L.G.A. of Edo State, Nigeria. The figure recorded in their study of $47.5 \%$ compares favourably with the $48.6 \%$ prevalence reported by Edungbola and Asaolu [11] in Kwara State, Nigeria, but higher than the $26.9 \%$ recorded by Nwaorgu et al. [12] in an onchocerciasis mesoendemic area of Enugu State, Nigeria. However, Akinbo and Okaka [13] reported a high overall prevalence (83\%) of the disease in Ovia North East L.G.A., Edo State, Nigeria [14]. These reports suggest that we need a well-thought mathematical formulation of a simple and appropriate model for the spread and control of this disease.

\section{Model Formulation}

In this section, we define the parameters used in the model below as follows: $\beta_{1}$ is the contact rate between $H_{S}$ and $H_{E}$; $\beta_{2}$ is the contact rate between $H_{S}$ and $H_{I} ; \beta_{3}$ is the contact rate between $H_{E}$ and $H_{I} ; \beta_{4}$ is the contact rate between $H_{I}$

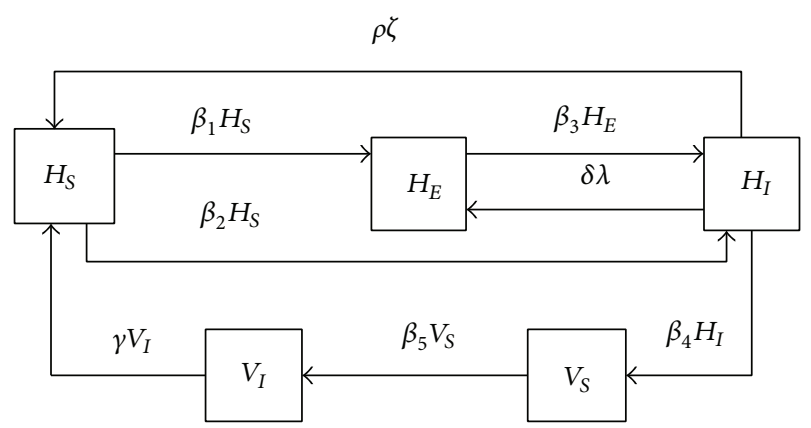

FIgURE 2: Model transfer diagram for onchocerciasis.

and $V_{S} ; \beta_{5}$ is the contact rate between $V_{S}$ and $V_{I} ; \gamma$ is the transmission rate from $V_{I}$ to $H_{S} ; \rho$ and $\delta$ are the proportions of Ivermectin/Mectizan treatment that cured the patient; $\zeta$ is the rate of movement of humans from the infective $H_{I}$ compartment into the human susceptible $H_{S}$ compartment; $\lambda$ is the rate of movement of human from the human infective $H_{I}$ compartment into the latent (incubation) period $H_{E}$ compartment.

\section{Model Diagram}

Figure 2 shows the model diagram which consists of five sets of ordinary differential equations highlighting the rate of change with respect to time of human susceptible compartment $H_{S}$; human latent (incubation) period compartment $H_{E}$; human infective compartment $H_{I}$; vector susceptible compartment $V_{S}$; and the vector infective compartment $V_{I}$. 


\section{Mathematical Formulation of the Model}

From the model transfer diagram (Figure 2), we have the following set of equations:

$$
\begin{gathered}
\frac{d H_{S}}{d t}=\rho \zeta+\gamma V_{I}-\left(\beta_{1}+\beta_{2}\right) H_{S}, \\
\frac{d H_{E}}{d t}=\delta \lambda+\beta_{1} H_{S}-\beta_{3} H_{E}, \\
\frac{d H_{I}}{d t}=\beta_{2} H_{S}+\beta_{3} H_{E}-\rho \zeta-\delta \lambda-\beta_{4} H_{I}, \\
\frac{d V_{S}}{d t}=\beta_{4} H_{I}-\beta_{5} V_{S}, \\
\frac{d V_{I}}{d t}=\beta_{5} V_{S}-\gamma V_{I} .
\end{gathered}
$$

To find the steady state, we assume that the compartments $V_{S}$, $V_{I}, H_{E}, H_{S}$, and $H_{I}$ do not depend on time. Therefore,

$$
\frac{d \overline{H_{S}}}{d t}=\frac{d \overline{H_{E}}}{d t}=\frac{d \overline{H_{I}}}{d t}=\frac{d \overline{V_{S}}}{d t}=\frac{d \overline{V_{I}}}{d t}=0 .
$$

We now solve the differential equations $d \overline{H_{S}} / d t=d \overline{H_{E}} / d t=$ $d \overline{H_{I}} / d t=d \overline{V_{S}} / d t=d \overline{V_{I}} / d t=0$ to find the value for $\overline{H_{S}}, \overline{H_{E}}$, $\overline{H_{I}}, \overline{V_{S}}$, and $\overline{V_{I}}$.

From (4),

$$
\begin{gathered}
\beta_{4} H_{I}-\beta_{5} V_{S}=0, \\
\overline{H_{I}}=\frac{\beta_{5}}{\beta_{4}} \overline{V_{S}}, \\
\overline{H_{I}}=A \overline{V_{S}} .
\end{gathered}
$$

From (5),

From (1),

$$
\begin{gathered}
\beta_{5} V_{S}-\gamma V_{I}=0, \\
\overline{V_{I}}=\frac{\beta_{5} \overline{V_{S}}}{\gamma}=B \overline{V_{S}} .
\end{gathered}
$$

$$
\begin{gathered}
\rho \zeta+\gamma V_{I}-\left(\beta_{1}+\beta_{2}\right) H_{S}=0 \\
\left(\beta_{1}+\beta_{2}\right) H_{S}=\rho \zeta+\gamma V_{I} .
\end{gathered}
$$

But

$$
\begin{gathered}
\overline{V_{I}}=\frac{\beta_{5} \overline{V_{S}}}{\gamma}, \\
\left(\beta_{1}+\beta_{2}\right) \overline{H_{S}}=\rho \zeta+\frac{\gamma \beta_{5} \overline{V_{S}}}{\gamma}, \\
\overline{H_{S}}=\frac{\rho \zeta+\beta_{5} \overline{V_{S}}}{\beta_{1}+\beta_{2}}=C \overline{V_{S}} .
\end{gathered}
$$

From (2),

$$
\begin{gathered}
\delta \lambda+\beta_{1} H_{S}-\beta_{3} H_{E}=0, \\
\beta_{3} \overline{H_{E}}=\delta \lambda+\beta_{1} \overline{H_{S}}, \\
\text { but, } \overline{H_{S}}=\frac{\rho \zeta+\beta_{5} \overline{V_{S}}}{\beta_{1}+\beta_{2}},
\end{gathered}
$$

$$
\begin{gathered}
\beta_{3} \overline{H_{E}}=\delta \lambda+\beta_{1}\left(\frac{\rho \zeta+\beta_{5} \overline{V_{S}}}{\beta_{1}+\beta_{2}}\right), \\
\overline{H_{E}}=\frac{\delta \lambda\left(\beta_{1}+\beta_{2}\right)+\beta_{1} \rho \zeta+\beta_{1} \beta_{5} \overline{V_{S}}}{\beta_{3}}=D \overline{V_{S}} .
\end{gathered}
$$

Lastly, from (3),

$$
\begin{gathered}
\beta_{2} H_{S}+\beta_{3} H_{E}-\rho \zeta-\delta \lambda-\beta_{4} H_{I}=0, \\
\beta_{2} \overline{H_{S}}+\beta_{3} \overline{H_{E}}-\rho \zeta-\delta \lambda-\beta_{4} \overline{H_{I}}=0, \\
\beta_{2} C \overline{V_{S}}+\beta_{3} D \overline{V_{S}}-\rho \zeta-\delta \lambda-\beta_{4} A \overline{V_{S}}=0 .
\end{gathered}
$$

We have that

$$
\overline{V_{S}}=\frac{\rho \zeta+\delta \lambda}{\beta_{2} C+\beta_{3} D-\beta_{4} A},
$$

provided that $\beta_{2} C+\beta_{3} D>\beta_{4} A$.

Therefore, the disease free equilibrium (DFE) is given as

$$
E_{0}=\left(\frac{\rho \zeta}{\beta_{1}+\beta_{2}}, 0,0,0,0\right)
$$

and the endemic equilibrium is given as

$$
\overline{H_{S}}=\frac{\rho \zeta+\beta_{5}}{\beta_{1}+\beta_{2}}((\rho \zeta+\delta \lambda)
$$

$$
\begin{aligned}
\times\left(\beta_{2}\left(\frac{\rho \zeta+\beta_{5}}{\beta_{1}+\beta_{2}}\right)+\beta_{3}\right. \\
\times\left(\frac{\delta \lambda\left(\beta_{1}+\beta_{2}\right)+\beta_{1} \rho \zeta+\beta_{1} \beta_{5}}{\beta_{3}}\right) \\
\left.\left.\quad-\beta_{4}\left(\frac{\beta_{5}}{\beta_{4}}\right)\right)^{-1}\right)
\end{aligned}
$$

$$
=\left(\left(\rho \zeta+\beta_{5}\right)(\rho \zeta+\delta \lambda)\right)
$$$$
\times\left(\beta_{2}\left(\rho \zeta+\beta_{5}\right)+\left(\beta_{1}+\beta_{2}\right)\right.
$$$$
\times\left[\delta \lambda\left(\beta_{1}+\beta_{2}\right)+\beta_{1} \rho \zeta+\beta_{1} \beta_{5}\right]
$$$$
\left.-\left(\beta_{1}+\beta_{2}\right) \beta_{5}\right)^{-1} \text {, }
$$

$$
\overline{H_{E}}=\frac{\delta \lambda\left(\beta_{1}+\beta_{2}\right)+\beta_{1} \rho \zeta+\beta_{1} \beta_{5}}{\beta_{3}}
$$

$$
\begin{gathered}
\times\left(( \rho \zeta + \delta \lambda ) \left(\beta_{2}\left(\frac{\rho \zeta+\beta_{5}}{\beta_{1}+\beta_{2}}\right)+\delta \lambda\left(\beta_{1}+\beta_{2}\right)\right.\right. \\
\left.\left.+\beta_{1} \rho \zeta+\beta_{1} \beta_{5}-\beta_{5}\right)^{-1}\right)
\end{gathered}
$$




$$
\begin{gathered}
=\left(\left[\delta \lambda\left(\beta_{1}+\beta_{2}\right)+\beta_{1} \rho \zeta+\beta_{1} \beta_{5}\right](\rho \zeta+\delta \lambda)\right) \\
\times\left(\frac{\left(\beta_{2} \beta_{3}\right)\left(\rho \zeta+\beta_{5}\right)}{\beta_{1}+\beta_{2}}+\delta \lambda\left(\beta_{1}+\beta_{2}\right) \beta_{3}\right. \\
\left.+\beta_{1} \beta_{3} \rho \zeta+\beta_{1} \beta_{3} \beta_{5}-\beta_{3} \beta_{5}\right)^{-1}, \\
\overline{H_{I}}=\frac{\beta_{5}}{\beta_{4}}((\rho \zeta+\delta \lambda) \\
\times\left(\beta_{2}\left(\frac{\rho \zeta+\beta_{5}}{\beta_{1}+\beta_{2}}\right)\right. \\
+\beta_{3}\left(\frac{\delta \lambda\left(\beta_{1}+\beta_{2}\right)+\beta_{1} \rho \zeta+\beta_{1} \beta_{5}}{\beta_{3}}\right) \\
\left.\left.-\beta_{4}\left(\frac{\beta_{5}}{\beta_{4}}\right)\right)^{-1}\right) \\
=\left(\beta_{5}(\rho \zeta+\delta \lambda)\right)\left(\beta_{2} \beta_{4}\left(\frac{\rho \zeta+\beta_{5}}{\beta_{1}+\beta_{2}}\right)+\beta_{4} \delta \lambda\left(\beta_{1}+\beta_{2}\right)\right. \\
\left.+\beta_{1} \beta_{4} \rho \zeta+\beta_{1} \beta_{4} \beta_{5}-\beta_{4} \beta_{5}\right)^{-1},
\end{gathered}
$$$$
\overline{V_{S}}=((\rho \zeta+\delta \lambda)
$$$$
\times\left(\beta_{2}\left(\frac{\rho \zeta+\beta_{5}}{\beta_{1}+\beta_{2}}\right)\right.
$$$$
+\beta_{3}\left(\frac{\delta \lambda\left(\beta_{1}+\beta_{2}\right)+\beta_{1} \rho \zeta+\beta_{1} \beta_{5}}{\beta_{3}}\right)
$$$$
\left.\left.-\beta_{4}\left(\frac{\beta_{5}}{\beta_{4}}\right)\right)^{-1}\right) \text {, }
$$

$$
\begin{aligned}
\overline{V_{I}}=\frac{\beta_{5}}{\gamma}( & (\rho \zeta+\delta \lambda) \\
& \times\left(\beta_{2}\left(\frac{\rho \zeta+\beta_{5}}{\beta_{1}+\beta_{2}}\right)\right. \\
& +\beta_{3}\left(\frac{\delta \lambda\left(\beta_{1}+\beta_{2}\right)+\beta_{1} \rho \zeta+\beta_{1} \beta_{5}}{\beta_{3}}\right) \\
=\left(\beta_{5}(\rho \zeta+\delta \lambda)\right) & \\
\left.\times\left(\gamma \beta_{4}\left(\frac{\rho \zeta+\beta_{5}}{\beta_{4}}\right)\right)^{-1}\right) & \\
& \left.+\gamma \beta_{1} \rho \zeta+\gamma \beta_{2} \beta_{5}-\gamma \beta_{5}\right)^{-1} .
\end{aligned}
$$

\section{Stability Analysis}

To assess the stability of the (DFE), we solve the model characteristics equation using the Jacobean matrix formulated from (1), (2), (3), (4), and (5) above as follows:

$$
J=\left(\begin{array}{ccccc}
-\left(\beta_{1}+\beta_{2}\right) & 0 & 0 & 0 & \gamma \\
\beta_{1} & -\beta_{3} & 0 & 0 & 0 \\
\beta_{2} & \beta_{3} & -\beta_{4} & 0 & 0 \\
0 & 0 & \beta_{4} & -\beta_{5} & 0 \\
0 & 0 & 0 & \beta_{5} & -\gamma
\end{array}\right)
$$

Applying the eigenvalue

$$
\lambda: \operatorname{det}(J-\lambda I)=0,
$$

we have

$$
\begin{aligned}
& \operatorname{det}\left[\begin{array}{ccccc}
-\left(\beta_{1}+\beta_{2}\right)-\lambda & 0 & 0 & 0 & \gamma \\
\beta_{1} & -\beta_{3}-\lambda & 0 & 0 & 0 \\
\beta_{2} & \beta_{3} & -\beta_{4}-\lambda & 0 & 0 \\
0 & 0 & \beta_{4} & -\beta_{5}-\lambda & 0 \\
0 & 0 & 0 & \beta_{5} & -\gamma-\lambda
\end{array}\right]=0 \\
& \operatorname{det}\left[\begin{array}{ccccc}
-\left(\beta_{1}+\beta_{2}+\lambda\right) & 0 & 0 & 0 & \gamma \\
\beta_{1} & -\left(\beta_{3}+\lambda\right) & 0 & 0 & 0 \\
\beta_{2} & \beta_{3} & -\left(\beta_{4}+\lambda\right) & 0 & 0 \\
0 & 0 & \beta_{4} & -\left(\beta_{5}+\lambda\right) & 0 \\
0 & 0 & 0 & \beta_{5} & -(\gamma+\lambda)
\end{array}\right]=0 \text {, } \\
& -\left(\beta_{1}+\beta_{2}+\lambda\right)\left[\begin{array}{cccc}
-\left(\beta_{3}+\lambda\right) & 0 & 0 & 0 \\
\beta_{3} & -\left(\beta_{4}+\lambda\right) & 0 & 0 \\
0 & \beta_{4} & -\left(\beta_{5}+\lambda\right) & 0 \\
0 & 0 & \beta_{5} & -(\gamma+\lambda)
\end{array}\right]+\gamma\left[\begin{array}{cccc}
\beta_{1}-\left(\beta_{3}+\lambda\right) & 0 & 0 \\
\beta_{2} & \beta_{3} & -\left(\beta_{4}+\lambda\right) & 0 \\
0 & 0 & \beta_{4} & -\left(\beta_{5}+\lambda\right) \\
0 & 0 & 0 & \beta_{5}
\end{array}\right]=0 \text {, }
\end{aligned}
$$




$$
\begin{gathered}
-\left(\beta_{1}+\beta_{2}+\lambda\right)\left[-\left(\beta_{3}+\lambda\right)\left|\begin{array}{ccc}
-\left(\beta_{4}+\lambda\right) & 0 & 0 \\
\beta_{4} & -\left(\beta_{5}+\lambda\right) & 0 \\
0 & \beta_{5} & -(\gamma+\lambda)
\end{array}\right|\right] \\
+\gamma\left[\beta_{1}\left[\begin{array}{ccc}
\beta_{3}-\left(\beta_{4}+\lambda\right) & 0 \\
0 & \beta_{4} & -\left(\beta_{5}+\lambda\right) \\
0 & 0 & \beta_{5}
\end{array}\right]+\left(\beta_{3}+\lambda\right)\left[\begin{array}{ccc}
\beta_{2} & -\left(\beta_{4}+\lambda\right) & 0 \\
0 & \beta_{4} & -\left(\beta_{5}+\lambda\right) \\
0 & 0 & \beta_{5}
\end{array}\right]\right]=0, \\
-\left(\beta_{1}+\beta_{2}+\lambda\right)\left[-\left(\beta_{3}+\lambda\right)\left[-\left(\beta_{4}+\lambda\right) \mid \begin{array}{ccc}
-\left(\beta_{5}+\lambda\right) & 0 & \mid \\
\beta_{5} & -(\gamma+\lambda) \mid
\end{array}\right]+\gamma \beta_{1} \beta_{3} \beta_{4} \beta_{5}+\gamma\left(\beta_{3}+\lambda\right) \beta_{2} \beta_{4} \beta_{5}=0,\right. \\
-\left[\left(\beta_{1}+\beta_{2}+\lambda\right)\left(\beta_{3}+\lambda\right)\left(\beta_{4}+\lambda\right)\left(\beta_{5}+\lambda\right)(\gamma+\lambda)\right]+\gamma \beta_{1} \beta_{3} \beta_{4} \beta_{5}+\gamma \beta_{2} \beta_{3} \beta_{4} \beta_{5}+\gamma \lambda \beta_{2} \beta_{4} \beta_{5}=0 .
\end{gathered}
$$

Hence, we obtain from $-\left(\beta_{1}+\beta_{2}+\lambda\right)=0$

$$
\lambda_{1}=-\left(\beta_{1}+\beta_{2}\right) \text {. }
$$

From $-\left(\beta_{3}+\lambda\right)=0$,

$$
\lambda_{2}=-\beta_{3} \text {. }
$$

From $-\left(\beta_{4}+\lambda\right)=0$,

$$
\lambda_{3}=-\beta_{4}
$$

From $\left(\beta_{5}+\lambda\right)=0$,

$$
\lambda_{4}=-\beta_{5} .
$$

From $(\gamma+\lambda)=0$

$$
\lambda_{5}=-\gamma
$$

Given that the results of $\lambda_{1}, \lambda_{2}, \lambda_{3}, \lambda_{4}$, and $\lambda_{5}$ are all negative and considering the principles of linearized stability which says that the systems are asymptotically stable if and only if all the eigenvalues of the system have negative real parts, we say that the disease-free equilibrium (DFE) is asymptotically stable.

\section{Application of the Homotopy Decomposition Method (HDM)}

In this section, we apply the HDM [15-17] to solve these mathematical sets of equations representing the onchocerciasis disease model as follows:

$$
\begin{gathered}
H_{S}(t)-H_{S}(0)=\int_{0}^{t}\left(\rho \zeta+\gamma V_{I}(\tau)-\left(\beta_{1}+\beta_{2}\right) H_{S}(\tau)\right) d \tau, \\
H_{E}(t)-H_{E}(0)=\int_{0}^{t}\left(\delta \lambda+\beta_{1} H_{S}(\tau)-\beta_{3} H_{E}(\tau)\right) d \tau,
\end{gathered}
$$

$$
H_{I}(t)-H_{I}(0)
$$$$
=\int_{0}^{t}\left(\beta_{2} H_{S}(\tau)+\beta_{3} H_{E}(\tau)-\rho \zeta-\delta \lambda-\beta_{4} H_{I}(\tau)\right) d \tau,
$$

$$
\begin{aligned}
& V_{S}(t)-V_{S}(0)=\int_{0}^{t}\left(\beta_{4} H_{I}(\tau)-\beta_{5} V_{S}(\tau)\right) d \tau, \\
& V_{I}(t)-V_{I}(0)=\int_{0}^{t}\left(\beta_{5} V_{S}(\tau)-\gamma V_{I}(\tau)\right) d \tau .
\end{aligned}
$$

We then assume that the solutions of the above integral equations can be put in the following form, for $P \in(0,1)$,

$$
\begin{gathered}
H_{S}(t)=\sum_{n=0}^{\infty} P^{n} H_{S n}(t), \quad H_{E}(t)=\sum_{n=0}^{\infty} P^{n} H_{E n}(t), \\
H_{I}(t)=\sum_{n=0}^{\infty} P^{n} H_{I n}(t), \quad V_{S}(t)=\sum_{n=0}^{\infty} P^{n} V_{S n}(t), \\
V_{I}(t)=\sum_{n=0}^{\infty} P^{n} V_{I n}(t) .
\end{gathered}
$$

Replacing the above expressions in the integral equations, we obtain the following expressions:

$$
\begin{aligned}
& \sum_{n=0}^{\infty} P^{n} H_{S n}(t)-H_{S}(0) \\
&=P \int_{0}^{t}\left[\left(\rho \zeta+\gamma \sum_{n=0}^{\infty} P^{n} V_{\text {In }}(\tau)\right.\right.
\end{aligned}
$$




$$
\left.\left.-\left(\beta_{1}+\beta_{2}\right) \sum_{n=0}^{\infty} P^{n} H_{S n}(\tau)\right) d \tau\right]
$$

$\sum_{n=0}^{\infty} P^{n} H_{E n}(t)-H_{E}(0)$

$$
\begin{aligned}
=P \int_{0}^{t}[(\delta \lambda & +\beta_{1} \sum_{n=0}^{\infty} P^{n} H_{S n}(\tau) \\
& \left.\left.-\beta_{3} \sum_{n=0}^{\infty} P^{n} H_{E n}(\tau)\right) d \tau\right],
\end{aligned}
$$

$\sum_{n=0}^{\infty} P^{n} H_{I n}(t)-H_{I}(0)$

$$
\begin{aligned}
=P \int_{0}^{t}\left[\left(\beta_{2} \sum_{n=0}^{\infty} P^{n} H_{S n}(\tau)+\beta_{3} \sum_{n=0}^{\infty} P^{n} H_{E n}(\tau)\right.\right. \\
\left.\left.-\rho \zeta-\delta \lambda-\beta_{4} \sum_{n=0}^{\infty} P^{n} H_{I n}(\tau)\right) d \tau\right],
\end{aligned}
$$

$$
\begin{aligned}
& \sum_{n=0}^{\infty} P^{n} V_{S n}(t)-V_{S}(0) \\
& \quad=P \int_{0}^{t}\left[\left(\beta_{4} \sum_{n=0}^{\infty} P^{n} H_{I n}(\tau)-\beta_{5} \sum_{n=0}^{\infty} P^{n} V_{S n}(\tau)\right) d \tau\right],
\end{aligned}
$$

$$
\begin{aligned}
& \sum_{n=0}^{\infty} P^{n} V_{I n}(t)-V_{I}(0) \\
& \quad=P \int_{0}^{t}\left[\left(\beta_{5} \sum_{n=0}^{\infty} P^{n} V_{S n}(\tau)-\gamma \sum_{n=0}^{\infty} P^{n} V_{I n}(\tau)\right) d \tau\right] .
\end{aligned}
$$

We compared the terms of the same power of $P$ and obtained the following:

$$
\begin{gathered}
P^{0}: H_{S 0}(t)=H_{S}(0), \\
P^{0}: H_{E 0}(t)=H_{E}(0), \\
P^{0}: H_{I 0}(t)=H_{I}(0), \\
P^{0}: V_{S 0}(t)=V_{S}(0), \\
P^{0}: V_{I 0}(t)=V_{I}(0), \\
P^{1}: H_{S 1}(t)=\int_{0}^{t}\left(\rho \zeta+\gamma V_{I 0}(\tau)-\left(\beta_{1}+\beta_{2}\right) H_{S 0}(\tau)\right) d \tau, \\
P^{1}: H_{E 1}(t)=\int_{0}^{t}\left(\delta \lambda+\beta_{1} H_{S 0}(\tau)-\beta_{3} H_{E 0}(\tau)\right) d \tau, \\
P^{1}: H_{I 1}(t) \quad \\
=\int_{0}^{t}\left(\beta_{2} H_{S 0}(\tau)+\beta_{3} H_{E 0}(\tau)-\rho \zeta-\delta \lambda-\beta_{4} H_{I 0}(\tau)\right) d \tau, \\
P^{1}: V_{S 1}(t)=\int_{0}^{t}\left(\beta_{4} H_{I 0}(\tau)-\beta_{5} V_{S 0}(\tau)\right) d \tau, \\
P^{1}: V_{I 1}(t)=\int_{0}^{t}\left(\beta_{5} V_{S 0}(\tau)-\gamma V_{I 0}(\tau)\right) d \tau .
\end{gathered}
$$

Continuing for $n \geq 2$, we have

$$
\begin{gathered}
H_{S n}(t)=\int_{0}^{t}\left(\gamma V_{I(n-1)}(\tau)-\left(\beta_{1}+\beta_{2}\right) H_{S(n-1)}(\tau)\right) d \tau, \\
H_{E n}(t)=\int_{0}^{t}\left(\beta_{1} H_{S(n-1)}(\tau)-\beta_{3} H_{E(n-1)}(\tau)\right) d \tau, \\
H_{I n}(t)=\int_{0}^{t}\left(\beta_{2} H_{S(n-1)}(\tau)+\beta_{3} H_{E(n-1)}(\tau)\right. \\
\left.\quad-\beta_{4} H_{I(n-1)}(\tau)\right) d \tau, \\
V_{S n}(t)=\int_{0}^{t}\left(\beta_{4} H_{I(n-1)}(\tau)-\beta_{5} V_{S(n-1)}(\tau)\right) d \tau, \\
V_{I n}(t)=\int_{0}^{t}\left(\beta_{5} V_{S(n-1)}(\tau)-\gamma V_{I(n-1)}(\tau)\right) d \tau .
\end{gathered}
$$

We now integrate the above equations to obtain the following:

$$
\begin{aligned}
& H_{I 0}(t)=0, \quad H_{E 0}(t)=0, \quad H_{S 0}[t]=N, \quad V_{S 0}[t]=M, \\
& V_{I 0}[t]=0, \quad H_{I 1}(t)=t\left(-\delta \lambda-\zeta \rho+N \beta_{2}\right), \\
& H_{S 1}[t]=t\left(\zeta \rho-N\left(\beta_{1}+\beta_{2}\right)\right), \\
& H_{E 1}(t)=t\left(\delta \lambda+N \beta_{1}\right) \text {, } \\
& V_{I 1}[t]=M t \beta_{5}, \quad V_{S 1}[t]=-M t \beta_{5}, \\
& H_{I 2}(t)=\frac{1}{2} t^{2} \zeta \rho \beta_{2}-\frac{1}{2} N t^{2} \beta_{1} \beta_{2}-\frac{1}{2} N t^{2} \beta_{2}^{2}+\frac{1}{2} t^{2} \delta \lambda \beta_{3} \\
& +\frac{1}{2} N t^{2} \beta_{1} \beta_{3}+\frac{1}{2} t^{2} \delta \lambda \beta_{4}+\frac{1}{2} t^{2} \zeta \rho \beta_{4}-\frac{1}{2} N t^{2} \beta_{2} \beta_{4}, \\
& H_{E 2}(t)=\frac{1}{2} t^{2} \zeta \rho \beta_{1}-\frac{1}{2} N t^{2} \beta_{1}^{2}-\frac{1}{2} N t^{2} \beta_{1} \beta_{2} \\
& -\frac{1}{2} t^{2} \delta \lambda \beta_{3}-\frac{1}{2} N t^{2} \beta_{1} \beta_{3} \\
& H_{S 2}[t]=-\frac{1}{2} t^{2} \zeta \rho \beta_{1}+\frac{1}{2} N t^{2} \beta_{1}^{2}-\frac{1}{2} t^{2} \zeta \rho \beta_{2} \\
& +N t^{2} \beta_{1} \beta_{2}+\frac{1}{2} N t^{2} \beta_{2}^{2}+\frac{1}{2} M t^{2} \gamma \beta_{5}, \\
& V_{S 2}[t]=-\frac{1}{2} t^{2} \delta \lambda \beta_{4}-\frac{1}{2} t^{2} \zeta \rho \beta_{4} \\
& +\frac{1}{2} N t^{2} \beta_{2} \beta_{4}+\frac{1}{2} M t^{2} \beta_{5}^{2}, \\
& V_{I 2}[t]=-\frac{1}{2} M t^{2} \gamma \beta_{5}-\frac{1}{2} M t^{2} \beta_{5}^{2} \text {, } \\
& H_{I 3}(t)=-\frac{1}{6} t^{3} \zeta \rho \beta_{1} \beta_{2}+\frac{1}{6} N t^{3} \beta_{1}^{2} \beta_{2}-\frac{1}{6} t^{3} \zeta \rho \beta_{2}^{2} \\
& +\frac{1}{3} N t^{3} \beta_{1} \beta_{2}^{2}+\frac{1}{6} N t^{3} \beta_{2}^{3}+\frac{1}{6} t^{3} \zeta \rho \beta_{1} \beta_{3} \\
& -\frac{1}{6} N t^{3} \beta_{1}^{2} \beta_{3}-\frac{1}{6} N t^{3} \beta_{1} \beta_{2} \beta_{3} \\
& -\frac{1}{6} t^{3} \delta \lambda \beta_{3}^{2} \\
& -\frac{1}{6} N t^{3} \beta_{1} \beta_{3}^{2}-\frac{1}{6} t^{3} \zeta \rho \beta_{2} \beta_{4} \\
& +\frac{1}{6} N t^{3} \beta_{1} \beta_{2} \beta_{4}+\frac{1}{6} N t^{3} \beta_{2}^{2} \beta_{4} \\
& -\frac{1}{6} t^{3} \delta \lambda \beta_{3} \beta_{4}-\frac{1}{6} N t^{3} \beta_{1} \beta_{3} \beta_{4}
\end{aligned}
$$




$$
\begin{aligned}
& -\frac{1}{6} t^{3} \delta \lambda \beta_{4}^{2}-\frac{1}{6} t^{3} \zeta \rho \beta_{4}^{2}+\frac{1}{6} N t^{3} \beta_{2} \beta_{4}^{2} \\
& +\frac{1}{6} M t^{3} \gamma \beta_{2} \beta_{5} \text {, } \\
& H_{E 3}(t)=-\frac{1}{6} t^{3} \zeta \rho \beta_{1}^{2}+\frac{1}{6} N t^{3} \beta_{1}^{3}-\frac{1}{6} t^{3} \zeta \rho \beta_{1} \beta_{2} \\
& +\frac{1}{3} N t^{3} \beta_{1}^{2} \beta_{2}+\frac{1}{6} N t^{3} \beta_{1} \beta_{2}^{2} \\
& -\frac{1}{6} t^{3} \zeta \rho \beta_{1} \beta_{3}+\frac{1}{6} N t^{3} \beta_{1}^{2} \beta_{3} \\
& +\frac{1}{6} N t^{3} \beta_{1} \beta_{2} \beta_{3}+\frac{1}{6} t^{3} \delta \lambda \beta_{3}^{2} \\
& +\frac{1}{6} N t^{3} \beta_{1} \beta_{3}^{2}+\frac{1}{6} M t^{3} \gamma \beta_{1} \beta_{5} \text {, } \\
& H_{S 3}[t]=\frac{1}{6} t^{3} \zeta \rho \beta_{1}^{2}-\frac{1}{6} N t^{3} \beta_{1}^{3}+\frac{1}{3} t^{3} \zeta \rho \beta_{1} \beta_{2} \\
& -\frac{1}{2} N t^{3} \beta_{1}^{2} \beta_{2}+\frac{1}{6} t^{3} \zeta \rho \beta_{2}^{2}-\frac{1}{2} N t^{3} \beta_{1} \beta_{2}^{2} \\
& -\frac{1}{6} N t^{3} \beta_{2}^{3}-\frac{1}{6} M t^{3} \gamma^{2} \beta_{5} \\
& -\frac{1}{6} M t^{3} \gamma \beta_{1} \beta_{5} \\
& -\frac{1}{6} M t^{3} \gamma \beta_{2} \beta_{5}-\frac{1}{6} M t^{3} \gamma \beta_{5}^{2}, \\
& V_{S 3}[t]=\frac{1}{6} t^{3} \zeta \rho \beta_{2} \beta_{4}-\frac{1}{6} N t^{3} \beta_{1} \beta_{2} \beta_{4}-\frac{1}{6} N t^{3} \beta_{2}^{2} \beta_{4} \\
& +\frac{1}{6} t^{3} \delta \lambda \beta_{3} \beta_{4}+\frac{1}{6} N t^{3} \beta_{1} \beta_{3} \beta_{4}+\frac{1}{6} t^{3} \delta \lambda \beta_{4}^{2} \\
& +\frac{1}{6} t^{3} \zeta \rho \beta_{4}^{2}-\frac{1}{6} N t^{3} \beta_{2} \beta_{4}^{2}+\frac{1}{6} t^{3} \delta \lambda \beta_{4} \beta_{5} \\
& +\frac{1}{6} t^{3} \zeta \rho \beta_{4} \beta_{5}-\frac{1}{6} N t^{3} \beta_{2} \beta_{4} \beta_{5}-\frac{1}{6} M t^{3} \beta_{5}^{3} \text {, } \\
& V_{I 3}[t]=\frac{1}{6} M t^{3} \gamma^{2} \beta_{5}-\frac{1}{6} t^{3} \delta \lambda \beta_{4} \beta_{5}-\frac{1}{6} t^{3} \zeta \rho \beta_{4} \beta_{5} \\
& +\frac{1}{6} N t^{3} \beta_{2} \beta_{4} \beta_{5}+\frac{1}{6} M t^{3} \gamma \beta_{5}^{2}+\frac{1}{6} M t^{3} \beta_{5}^{3} \text {, } \\
& H_{S 4}[t]=-\frac{1}{24} t^{4} \zeta \rho \beta_{1}^{3}+\frac{1}{24} N t^{4} \beta_{1}^{4}-\frac{1}{8} t^{4} \zeta \rho \beta_{1}^{2} \beta_{2} \\
& +\frac{1}{6} N t^{4} \beta_{1}^{3} \beta_{2}-\frac{1}{8} t^{4} \zeta \rho \beta_{1} \beta_{2}^{2}+\frac{1}{4} N t^{4} \beta_{1}^{2} \beta_{2}^{2} \\
& -\frac{1}{24} t^{4} \zeta \rho \beta_{2}^{3}+\frac{1}{6} N t^{4} \beta_{1} \beta_{2}^{3}+\frac{1}{24} N t^{4} \beta_{2}^{4} \\
& +\frac{1}{24} M t^{4} \gamma^{3} \beta_{5}+\frac{1}{24} M t^{4} \gamma^{2} \beta_{1} \beta_{5} \\
& +\frac{1}{24} M t^{4} \gamma \beta_{1}^{2} \beta_{5}+\frac{1}{24} M t^{4} \gamma^{2} \beta_{2} \beta_{5} \\
& +\frac{1}{12} M t^{4} \gamma \beta_{1} \beta_{2} \beta_{5}+\frac{1}{24} M t^{4} \gamma \beta_{2}^{2} \beta_{5} \\
& -\frac{1}{24} t^{4} \gamma \delta \lambda \beta_{4} \beta_{5}-\frac{1}{24} t^{4} \gamma \zeta_{\rho} \beta_{4} \beta_{5} \\
& +\frac{1}{24} N t^{4} \gamma \beta_{2} \beta_{4} \beta_{5}+\frac{1}{24} M t^{4} \gamma^{2} \beta_{5}^{2} \\
& +\frac{1}{24} M t^{4} \gamma \beta_{1} \beta_{5}^{2}+\frac{1}{24} M t^{4} \gamma \beta_{2} \beta_{5}^{2}+\frac{1}{24} M t^{4} \gamma \beta_{5}^{3} \text {, } \\
& H_{E 4}(t)=\frac{1}{24} t^{4} \zeta \rho \beta_{1}^{3}-\frac{1}{24} N t^{4} \beta_{1}^{4}+\frac{1}{12} t^{4} \zeta \rho \beta_{1}^{2} \beta_{2} \\
& -\frac{1}{8} N t^{4} \beta_{1}^{3} \beta_{2}+\frac{1}{24} t^{4} \zeta \rho \beta_{1} \beta_{2}^{2}-\frac{1}{8} N t^{4} \beta_{1}^{2} \beta_{2}^{2} \\
& -\frac{1}{24} N t^{4} \beta_{1} \beta_{2}^{3}+\frac{1}{24} t^{4} \zeta \rho \beta_{1}^{2} \beta_{3}-\frac{1}{24} N t^{4} \beta_{1}^{3} \beta_{3} \\
& +\frac{1}{24} t^{4} \zeta \rho \beta_{1} \beta_{2} \beta_{3}-\frac{1}{12} N t^{4} \beta_{1}^{2} \beta_{2} \beta_{3}-\frac{1}{24} N t^{4} \beta_{1} \beta_{2}^{2} \beta_{3} \\
& +\frac{1}{24} t^{4} \zeta \rho \beta_{1} \beta_{3}^{2}-\frac{1}{24} N t^{4} \beta_{1}^{2} \beta_{3}^{2}-\frac{1}{24} N t^{4} \beta_{1} \beta_{2} \beta_{3}^{2} \\
& -\frac{1}{24} t^{4} \delta \lambda \beta_{3}^{3}-\frac{1}{24} N t^{4} \beta_{1} \beta_{3}^{3}-\frac{1}{24} M t^{4} \gamma^{2} \beta_{1} \beta_{5} \\
& -\frac{1}{24} M t^{4} \gamma \beta_{1}^{2} \beta_{5}-\frac{1}{24} M t^{4} \gamma \beta_{1} \beta_{2} \beta_{5} \\
& -\frac{1}{24} M t^{4} \gamma \beta_{1} \beta_{3} \beta_{5}-\frac{1}{24} M t^{4} \gamma \beta_{1} \beta_{5}^{2} \text {, } \\
& H_{I 4}(t)=\frac{1}{24} t^{4} \zeta \rho \beta_{1}^{2} \beta_{2}-\frac{1}{24} N t^{4} \beta_{1}^{3} \beta_{2}+\frac{1}{12} t^{4} \zeta \rho \beta_{1} \beta_{2}^{2} \\
& -\frac{1}{8} N t^{4} \beta_{1}^{2} \beta_{2}^{2}+\frac{1}{24} t^{4} \zeta \rho \beta_{2}^{3}-\frac{1}{8} N t^{4} \beta_{1} \beta_{2}^{3} \\
& -\frac{1}{24} N t^{4} \beta_{2}^{4}-\frac{1}{24} t^{4} \zeta \rho \beta_{1}^{2} \beta_{3}+\frac{1}{24} N t^{4} \beta_{1}^{3} \beta_{3} \\
& -\frac{1}{24} t^{4} \zeta \rho \beta_{1} \beta_{2} \beta_{3}+\frac{1}{12} N t^{4} \beta_{1}^{2} \beta_{2} \beta_{3} \\
& +\frac{1}{24} N t^{4} \beta_{1} \beta_{2}^{2} \beta_{3} \\
& -\frac{1}{24} t^{4} \zeta \rho \beta_{1} \beta_{3}^{2}+\frac{1}{24} N t^{4} \beta_{1}^{2} \beta_{3}^{2}+\frac{1}{24} N t^{4} \beta_{1} \beta_{2} \beta_{3}^{2} \\
& +\frac{1}{24} t^{4} \delta \lambda \beta_{3}^{3}+\frac{1}{24} N t^{4} \beta_{1} \beta_{3}^{3}+\frac{1}{24} t^{4} \zeta \rho \beta_{1} \beta_{2} \beta_{4} \\
& -\frac{1}{24} N t^{4} \beta_{1}^{2} \beta_{2} \beta_{4}+\frac{1}{24} t^{4} \zeta \rho \beta_{2}^{2} \beta_{4}-\frac{1}{12} N t^{4} \beta_{1} \beta_{2}^{2} \beta_{4} \\
& -\frac{1}{24} N t^{4} \beta_{2}^{3} \beta_{4}-\frac{1}{24} t^{4} \zeta \rho \beta_{1} \beta_{3} \beta_{4} \\
& +\frac{1}{24} N t^{4} \beta_{1}^{2} \beta_{3} \beta_{4}+\frac{1}{24} N t^{4} \beta_{1} \beta_{2} \beta_{3} \beta_{4} \\
& +\frac{1}{24} t^{4} \delta \lambda \beta_{3}^{2} \beta_{4}+\frac{1}{24} N t^{4} \beta_{1} \beta_{3}^{2} \beta_{4} \\
& +\frac{1}{24} t^{4} \zeta \rho \beta_{2} \beta_{4}^{2}-\frac{1}{24} N t^{4} \beta_{1} \beta_{2} \beta_{4}^{2}-\frac{1}{24} N t^{4} \beta_{2}^{2} \beta_{4}^{2} \\
& +\frac{1}{24} t^{4} \delta \lambda \beta_{3} \beta_{4}^{2}+\frac{1}{24} N t^{4} \beta_{1} \beta_{3} \beta_{4}^{2}+\frac{1}{24} t^{4} \delta \lambda \beta_{4}^{3} \\
& +\frac{1}{24} t^{4} \zeta \rho \beta_{4}^{3}-\frac{1}{24} N t^{4} \beta_{2} \beta_{4}^{3}-\frac{1}{24} M t^{4} \gamma^{2} \beta_{2} \beta_{5} \\
& -\frac{1}{24} M t^{4} \gamma \beta_{1} \beta_{2} \beta_{5}-\frac{1}{24} M t^{4} \gamma \beta_{2}^{2} \beta_{5} \\
& +\frac{1}{24} M t^{4} \gamma \beta_{1} \beta_{3} \beta_{5} \\
& -\frac{1}{24} M t^{4} \gamma \beta_{2} \beta_{4} \beta_{5}-\frac{1}{24} M t^{4} \gamma \beta_{2} \beta_{5}^{2} \\
& V_{S 4}[t]=-\frac{1}{24} t^{4} \zeta \rho \beta_{1} \beta_{2} \beta_{4}+\frac{1}{24} N t^{4} \beta_{1}^{2} \beta_{2} \beta_{4} \\
& -\frac{1}{24} t^{4} \zeta \rho \beta_{2}^{2} \beta_{4}+\frac{1}{12} N t^{4} \beta_{1} \beta_{2}^{2} \beta_{4}
\end{aligned}
$$




$$
\begin{aligned}
& +\frac{1}{24} N t^{4} \beta_{2}^{3} \beta_{4}+\frac{1}{24} t^{4} \zeta \rho \beta_{1} \beta_{3} \beta_{4} \\
& -\frac{1}{24} N t^{4} \beta_{1}^{2} \beta_{3} \beta_{4}-\frac{1}{24} N t^{4} \beta_{1} \beta_{2} \beta_{3} \beta_{4} \\
& -\frac{1}{24} t^{4} \delta \lambda \beta_{3}^{2} \beta_{4} \\
& -\frac{1}{24} N t^{4} \beta_{1} \beta_{3}^{2} \beta_{4}-\frac{1}{24} t^{4} \zeta \rho \beta_{2} \beta_{4}^{2} \\
& +\frac{1}{24} N t^{4} \beta_{1} \beta_{2} \beta_{4}^{2}+\frac{1}{24} N t^{4} \beta_{2}^{2} \beta_{4}^{2} \\
& -\frac{1}{24} t^{4} \delta \lambda \beta_{3} \beta_{4}^{2}-\frac{1}{24} N t^{4} \beta_{1} \beta_{3} \beta_{4}^{2} \\
& -\frac{1}{24} t^{4} \delta \lambda \beta_{4}^{3}-\frac{1}{24} t^{4} \zeta \rho \beta_{4}^{3} \\
& +\frac{1}{24} N t^{4} \beta_{2} \beta_{4}^{3} \\
& +\frac{1}{24} M t^{4} \gamma \beta_{2} \beta_{4} \beta_{5}-\frac{1}{24} t^{4} \zeta \rho \beta_{2} \beta_{4} \beta_{5} \\
& +\frac{1}{24} N t^{4} \beta_{1} \beta_{2} \beta_{4} \beta_{5}+\frac{1}{24} N t^{4} \beta_{2}^{2} \beta_{4} \beta_{5} \\
& -\frac{1}{24} t^{4} \delta \lambda \beta_{3} \beta_{4} \beta_{5}-\frac{1}{24} N t^{4} \beta_{1} \beta_{3} \beta_{4} \beta_{5} \\
& -\frac{1}{24} t^{4} \delta \lambda \beta_{4}^{2} \beta_{5}-\frac{1}{24} t^{4} \zeta \rho \beta_{4}^{2} \beta_{5}+\frac{1}{24} N t^{4} \beta_{2} \beta_{4}^{2} \beta_{5} \\
& -\frac{1}{24} t^{4} \delta \lambda \beta_{4} \beta_{5}^{2}-\frac{1}{24} t^{4} \zeta \rho \beta_{4} \beta_{5}^{2} \\
& +\frac{1}{24} N t^{4} \beta_{2} \beta_{4} \beta_{5}^{2}+\frac{1}{24} M t^{4} \beta_{5}^{4}, \\
& V_{I 4}[t]=-\frac{1}{24} M t^{4} \gamma^{3} \beta_{5}+\frac{1}{24} t^{4} \gamma \delta \lambda \beta_{4} \beta_{5}+\frac{1}{24} t^{4} \gamma \zeta \rho \beta_{4} \beta_{5} \\
& -\frac{1}{24} N t^{4} \gamma \beta_{2} \beta_{4} \beta_{5}+\frac{1}{24} t^{4} \zeta \rho \beta_{2} \beta_{4} \beta_{5} \\
& -\frac{1}{24} N t^{4} \beta_{1} \beta_{2} \beta_{4} \beta_{5}-\frac{1}{24} N t^{4} \beta_{2}^{2} \beta_{4} \beta_{5} \\
& +\frac{1}{24} t^{4} \delta \lambda \beta_{3} \beta_{4} \beta_{5}+\frac{1}{24} N t^{4} \beta_{1} \beta_{3} \beta_{4} \beta_{5} \\
& +\frac{1}{24} t^{4} \delta \lambda \beta_{4}^{2} \beta_{5}+\frac{1}{24} t^{4} \zeta \rho \beta_{4}^{2} \beta_{5} \\
& -\frac{1}{24} N t^{4} \beta_{2} \beta_{4}^{2} \beta_{5}-\frac{1}{24} M t^{4} \gamma^{2} \beta_{5}^{2} \\
& +\frac{1}{24} t^{4} \delta \lambda \beta_{4} \beta_{5}^{2}+\frac{1}{24} t^{4} \zeta \rho \beta_{4} \beta_{5}^{2} \\
& -\frac{1}{24} N t^{4} \beta_{2} \beta_{4} \beta_{5}^{2}-\frac{1}{24} M t^{4} \gamma \beta_{5}^{3}-\frac{1}{24} M t^{4} \beta_{5}^{4}, \\
& H_{S 5}[t]=\frac{1}{120} t^{5} \zeta \rho \beta_{1}^{4}-\frac{1}{120} N t^{5} \beta_{1}^{5}+\frac{1}{30} t^{5} \zeta \rho \beta_{1}^{3} \beta_{2} \\
& -\frac{1}{24} N t^{5} \beta_{1}^{4} \beta_{2}+\frac{1}{20} t^{5} \zeta \rho \beta_{1}^{2} \beta_{2}^{2}-\frac{1}{12} N t^{5} \beta_{1}^{3} \beta_{2}^{2} \\
& +\frac{1}{30} t^{5} \zeta \rho \beta_{1} \beta_{2}^{3}-\frac{1}{12} N t^{5} \beta_{1}^{2} \beta_{2}^{3}+\frac{1}{120} t^{5} \zeta \rho \beta_{2}^{4} \\
& -\frac{1}{24} N t^{5} \beta_{1} \beta_{2}^{4}-\frac{1}{120} N t^{5} \beta_{2}^{5}-\frac{1}{120} M t^{5} \gamma^{4} \beta_{5} \\
& -\frac{1}{120} M t^{5} \gamma^{3} \beta_{1} \beta_{5}-\frac{1}{120} M t^{5} \gamma^{2} \beta_{1}^{2} \beta_{5}
\end{aligned}
$$

$$
\begin{aligned}
& -\frac{1}{120} M t^{5} \gamma \beta_{1}^{3} \beta_{5}-\frac{1}{120} M t^{5} \gamma^{3} \beta_{2} \beta_{5} \\
& -\frac{1}{60} M t^{5} \gamma^{2} \beta_{1} \beta_{2} \beta_{5}-\frac{1}{40} M t^{5} \gamma \beta_{1}^{2} \beta_{2} \beta_{5} \\
& -\frac{1}{120} M t^{5} \gamma^{2} \beta_{2}^{2} \beta_{5}-\frac{1}{40} M t^{5} \gamma \beta_{1} \beta_{2}^{2} \beta_{5} \\
& -\frac{1}{120} M t^{5} \gamma \beta_{2}^{3} \beta_{5}+\frac{1}{120} t^{5} \gamma^{2} \delta \lambda \beta_{4} \beta_{5} \\
& +\frac{1}{120} t^{5} \gamma^{2} \zeta \rho \beta_{4} \beta_{5}+\frac{1}{120} t^{5} \gamma \delta \lambda \beta_{1} \beta_{4} \beta_{5} \\
& +\frac{1}{120} t^{5} \gamma \zeta \rho \beta_{1} \beta_{4} \beta_{5}-\frac{1}{120} N t^{5} \gamma^{2} \beta_{2} \beta_{4} \beta_{5} \\
& +\frac{1}{120} t^{5} \gamma \delta \lambda \beta_{2} \beta_{4} \beta_{5}+\frac{1}{60} t^{5} \gamma \zeta \rho \beta_{2} \beta_{4} \beta_{5} \\
& -\frac{1}{60} N t^{5} \gamma \beta_{1} \beta_{2} \beta_{4} \beta_{5}-\frac{1}{60} N t^{5} \gamma \beta_{2}^{2} \beta_{4} \beta_{5} \\
& +\frac{1}{120} t^{5} \gamma \delta \lambda \beta_{3} \beta_{4} \beta_{5}+\frac{1}{120} N t^{5} \gamma \beta_{1} \beta_{3} \beta_{4} \beta_{5} \\
& +\frac{1}{120} t^{5} \gamma \delta \lambda \beta_{4}^{2} \beta_{5}+\frac{1}{120} t^{5} \gamma \zeta \rho \beta_{4}^{2} \beta_{5} \\
& -\frac{1}{120} N t^{5} \gamma \beta_{2} \beta_{4}^{2} \beta_{5}-\frac{1}{120} M t^{5} \gamma^{3} \beta_{5}^{2} \\
& -\frac{1}{120} M t^{5} \gamma^{2} \beta_{1} \beta_{5}^{2}-\frac{1}{120} M t^{5} \gamma \beta_{1}^{2} \beta_{5}^{2} \\
& -\frac{1}{120} M t^{5} \gamma^{2} \beta_{2} \beta_{5}^{2}-\frac{1}{60} M t^{5} \gamma \beta_{1} \beta_{2} \beta_{5}^{2} \\
& -\frac{1}{120} M t^{5} \gamma \beta_{2}^{2} \beta_{5}^{2}+\frac{1}{120} t^{5} \gamma \delta \lambda \beta_{4} \beta_{5}^{2} \\
& +\frac{1}{120} t^{5} \gamma \zeta \rho \beta_{4} \beta_{5}^{2}-\frac{1}{120} N t^{5} \gamma \beta_{2} \beta_{4} \beta_{5}^{2} \\
& -\frac{1}{120} M t^{5} \gamma^{2} \beta_{5}^{3}-\frac{1}{120} M t^{5} \gamma \beta_{1} \beta_{5}^{3} \\
& -\frac{1}{120} M t^{5} \gamma \beta_{2} \beta_{5}^{3}-\frac{1}{120} M t^{5} \gamma \beta_{5}^{4}, \\
& +
\end{aligned}
$$$$
H_{E 5}[t]=-\frac{1}{120} t^{5} \zeta \rho \beta_{1}^{4}+\frac{1}{120} N t^{5} \beta_{1}^{5}
$$$$
-\frac{1}{40} t^{5} \zeta \rho \beta_{1}^{3} \beta_{2}+\frac{1}{30} N t^{5} \beta_{1}^{4} \beta_{2}
$$$$
-\frac{1}{40} t^{5} \zeta \rho \beta_{1}^{2} \beta_{2}^{2}+\frac{1}{20} N t^{5} \beta_{1}^{3} \beta_{2}^{2}
$$$$
-\frac{1}{120} t^{5} \zeta \rho \beta_{1} \beta_{2}^{3}+\frac{1}{30} N t^{5} \beta_{1}^{2} \beta_{2}^{3}
$$$$
+\frac{1}{120} N t^{5} \beta_{1} \beta_{2}^{4}
$$$$
-\frac{1}{120} t^{5} \zeta \rho \beta_{1}^{3} \beta_{3}+\frac{1}{120} N t^{5} \beta_{1}^{4} \beta_{3}
$$$$
-\frac{1}{60} t^{5} \zeta \rho \beta_{1}^{2} \beta_{2} \beta_{3}+\frac{1}{40} N t^{5} \beta_{1}^{3} \beta_{2} \beta_{3}
$$$$
-\frac{1}{120} t^{5} \zeta \rho \beta_{1} \beta_{2}^{2} \beta_{3}+\frac{1}{40} N t^{5} \beta_{1}^{2} \beta_{2}^{2} \beta_{3}
$$$$
+\frac{1}{120} N t^{5} \beta_{1} \beta_{2}^{3} \beta_{3}-\frac{1}{120} t^{5} \zeta \rho \beta_{1}^{2} \beta_{3}^{2}
$$$$
+\frac{1}{120} N t^{5} \beta_{1}^{3} \beta_{3}^{2}-\frac{1}{120} t^{5} \zeta \rho \beta_{1} \beta_{2} \beta_{3}^{2}
$$ 


$$
\begin{aligned}
& +\frac{1}{60} N t^{5} \beta_{1}^{2} \beta_{2} \beta_{3}^{2}+\frac{1}{120} N t^{5} \beta_{1} \beta_{2}^{2} \beta_{3}^{2} \\
& +\frac{1}{40} N t^{5} \beta_{1} \beta_{2}^{3} \beta_{4}+\frac{1}{120} N t^{5} \beta_{2}^{4} \beta_{4} \\
& -\frac{1}{120} t^{5} \zeta \rho \beta_{1} \beta_{3}^{3}+\frac{1}{120} N t^{5} \beta_{1}^{2} \beta_{3}^{3} \\
& +\frac{1}{120} N t^{5} \beta_{1} \beta_{2} \beta_{3}^{3}+\frac{1}{120} t^{5} \delta \lambda \beta_{3}^{4} \\
& +\frac{1}{120} N t^{5} \beta_{1} \beta_{3}^{4}+\frac{1}{120} M t^{5} \gamma^{3} \beta_{1} \beta_{5} \\
& +\frac{1}{120} M t^{5} \gamma^{2} \beta_{1}^{2} \beta_{5}+\frac{1}{120} M t^{5} \gamma \beta_{1}^{3} \beta_{5} \\
& +\frac{1}{120} M t^{5} \gamma^{2} \beta_{1} \beta_{2} \beta_{5}+\frac{1}{60} M t^{5} \gamma \beta_{1}^{2} \beta_{2} \beta_{5} \\
& +\frac{1}{120} M t^{5} \gamma \beta_{1} \beta_{2}^{2} \beta_{5}+\frac{1}{120} M t^{5} \gamma^{2} \beta_{1} \beta_{3} \beta_{5} \\
& +\frac{1}{120} M t^{5} \gamma \beta_{1}^{2} \beta_{3} \beta_{5}+\frac{1}{120} M t^{5} \gamma \beta_{1} \beta_{2} \beta_{3} \beta_{5} \\
& +\frac{1}{120} M t^{5} \gamma \beta_{1} \beta_{3}^{2} \beta_{5}-\frac{1}{120} t^{5} \gamma \delta \lambda \beta_{1} \beta_{4} \beta_{5} \\
& -\frac{1}{120} t^{5} \gamma \zeta \rho \beta_{1} \beta_{4} \beta_{5}+\frac{1}{120} N t^{5} \gamma \beta_{1} \beta_{2} \beta_{4} \beta_{5} \\
& +\frac{1}{120} M t^{5} \gamma^{2} \beta_{1} \beta_{5}^{2}+\frac{1}{120} M t^{5} \gamma \beta_{1}^{2} \beta_{5}^{2} \\
& +\frac{1}{120} M t^{5} \gamma \beta_{1} \beta_{2} \beta_{5}^{2}+\frac{1}{120} M t^{5} \gamma \beta_{1} \beta_{3} \beta_{5}^{2} \\
& +\frac{1}{120} M t^{5} \gamma \beta_{1} \beta_{5}^{3} \text {, } \\
& H_{I 5}[t]=-\frac{1}{120} t^{5} \zeta \rho \beta_{1}^{3} \beta_{2}+\frac{1}{120} N t^{5} \beta_{1}^{4} \beta_{2}-\frac{1}{40} t^{5} \zeta \rho \beta_{1}^{2} \beta_{2}^{2} \\
& +\frac{1}{30} N t^{5} \beta_{1}^{3} \beta_{2}^{2}-\frac{1}{40} t^{5} \zeta \rho \beta_{1} \beta_{2}^{3}+\frac{1}{20} N t^{5} \beta_{1}^{2} \beta_{2}^{3} \\
& -\frac{1}{120} t^{5} \zeta \rho \beta_{2}^{4}+\frac{1}{30} N t^{5} \beta_{1} \beta_{2}^{4}+\frac{1}{120} N t^{5} \beta_{2}^{5} \\
& +\frac{1}{120} t^{5} \zeta \rho \beta_{1}^{3} \beta_{3}-\frac{1}{120} N t^{5} \beta_{1}^{4} \beta_{3} \\
& +\frac{1}{60} t^{5} \zeta \rho \beta_{1}^{2} \beta_{2} \beta_{3}-\frac{1}{40} N t^{5} \beta_{1}^{3} \beta_{2} \beta_{3} \\
& +\frac{1}{120} t^{5} \zeta \rho \beta_{1} \beta_{2}^{2} \beta_{3}-\frac{1}{40} N t^{5} \beta_{1}^{2} \beta_{2}^{2} \beta_{3} \\
& -\frac{1}{120} N t^{5} \beta_{1} \beta_{2}^{3} \beta_{3}+\frac{1}{120} t^{5} \zeta \rho \beta_{1}^{2} \beta_{3}^{2} \\
& -\frac{1}{120} N t^{5} \beta_{1}^{3} \beta_{3}^{2}+\frac{1}{120} t^{5} \zeta \rho \beta_{1} \beta_{2} \beta_{3}^{2} \\
& -\frac{1}{60} N t^{5} \beta_{1}^{2} \beta_{2} \beta_{3}^{2}-\frac{1}{120} N t^{5} \beta_{1} \beta_{2}^{2} \beta_{3}^{2} \\
& +\frac{1}{120} t^{5} \zeta \rho \beta_{1} \beta_{3}^{3}-\frac{1}{120} N t^{5} \beta_{1}^{2} \beta_{3}^{3} \\
& -\frac{1}{120} N t^{5} \beta_{1} \beta_{2} \beta_{3}^{3}-\frac{1}{120} t^{5} \delta \lambda \beta_{3}^{4} \\
& -\frac{1}{120} N t^{5} \beta_{1} \beta_{3}^{4}-\frac{1}{120} t^{5} \zeta \rho \beta_{1}^{2} \beta_{2} \beta_{4} \\
& +\frac{1}{120} N t^{5} \beta_{1}^{3} \beta_{2} \beta_{4}-\frac{1}{60} t^{5} \zeta \rho \beta_{1} \beta_{2}^{2} \beta_{4} \\
& +\frac{1}{40} N t^{5} \beta_{1}^{2} \beta_{2}^{2} \beta_{4}-\frac{1}{120} t^{5} \zeta \rho \beta_{2}^{3} \beta_{4} \\
& +\frac{1}{120} t^{5} \zeta \rho \beta_{1}^{2} \beta_{3} \beta_{4}-\frac{1}{120} N t^{5} \beta_{1}^{3} \beta_{3} \beta_{4} \\
& +\frac{1}{120} t^{5} \zeta \rho \beta_{1} \beta_{2} \beta_{3} \beta_{4}-\frac{1}{60} N t^{5} \beta_{1}^{2} \beta_{2} \beta_{3} \beta_{4} \\
& -\frac{1}{120} N t^{5} \beta_{1} \beta_{2}^{2} \beta_{3} \beta_{4}+\frac{1}{120} t^{5} \zeta \rho \beta_{1} \beta_{3}^{2} \beta_{4} \\
& -\frac{1}{120} N t^{5} \beta_{1}^{2} \beta_{3}^{2} \beta_{4}-\frac{1}{120} N t^{5} \beta_{1} \beta_{2} \beta_{3}^{2} \beta_{4} \\
& -\frac{1}{120} t^{5} \delta \lambda \beta_{3}^{3} \beta_{4}-\frac{1}{120} N t^{5} \beta_{1} \beta_{3}^{3} \beta_{4} \\
& -\frac{1}{120} t^{5} \zeta \rho \beta_{1} \beta_{2} \beta_{4}^{2}+\frac{1}{120} N t^{5} \beta_{1}^{2} \beta_{2} \beta_{4}^{2} \\
& -\frac{1}{120} t^{5} \zeta \rho \beta_{2}^{2} \beta_{4}^{2}+\frac{1}{60} N t^{5} \beta_{1} \beta_{2}^{2} \beta_{4}^{2} \\
& +\frac{1}{120} N t^{5} \beta_{2}^{3} \beta_{4}^{2}+\frac{1}{120} t^{5} \zeta \rho \beta_{1} \beta_{3} \beta_{4}^{2} \\
& -\frac{1}{120} N t^{5} \beta_{1}^{2} \beta_{3} \beta_{4}^{2}-\frac{1}{120} N t^{5} \beta_{1} \beta_{2} \beta_{3} \beta_{4}^{2} \\
& -\frac{1}{120} t^{5} \delta \lambda \beta_{3}^{2} \beta_{4}^{2}-\frac{1}{120} N t^{5} \beta_{1} \beta_{3}^{2} \beta_{4}^{2} \\
& -\frac{1}{120} t^{5} \zeta \rho \beta_{2} \beta_{4}^{3}+\frac{1}{120} N t^{5} \beta_{1} \beta_{2} \beta_{4}^{3} \\
& +\frac{1}{120} N t^{5} \beta_{2}^{2} \beta_{4}^{3}-\frac{1}{120} t^{5} \delta \lambda \beta_{3} \beta_{4}^{3} \\
& -\frac{1}{120} N t^{5} \beta_{1} \beta_{3} \beta_{4}^{3}-\frac{1}{120} t^{5} \delta \lambda \beta_{4}^{4} \\
& -\frac{1}{120} t^{5} \zeta \rho \beta_{4}^{4}+\frac{1}{120} N t^{5} \beta_{2} \beta_{4}^{4} \\
& +\frac{1}{120} M t^{5} \gamma^{3} \beta_{2} \beta_{5}+\frac{1}{120} M t^{5} \gamma^{2} \beta_{1} \beta_{2} \beta_{5} \\
& +\frac{1}{120} M t^{5} \gamma \beta_{1}^{2} \beta_{2} \beta_{5}+\frac{1}{120} M t^{5} \gamma^{2} \beta_{2}^{2} \beta_{5} \\
& +\frac{1}{60} M t^{5} \gamma \beta_{1} \beta_{2}^{2} \beta_{5}+\frac{1}{120} M t^{5} \gamma \beta_{2}^{3} \beta_{5} \\
& -\frac{1}{120} M t^{5} \gamma^{2} \beta_{1} \beta_{3} \beta_{5}-\frac{1}{120} M t^{5} \gamma \beta_{1}^{2} \beta_{3} \beta_{5} \\
& -\frac{1}{120} M t^{5} \gamma \beta_{1} \beta_{2} \beta_{3} \beta_{5}-\frac{1}{120} M t^{5} \gamma \beta_{1} \beta_{3}^{2} \beta_{5} \\
& +\frac{1}{120} M t^{5} \gamma^{2} \beta_{2} \beta_{4} \beta_{5}-\frac{1}{120} t^{5} \gamma \delta \lambda \beta_{2} \beta_{4} \beta_{5} \\
& -\frac{1}{120} t^{5} \gamma \zeta \rho \beta_{2} \beta_{4} \beta_{5}+\frac{1}{120} M t^{5} \gamma \beta_{1} \beta_{2} \beta_{4} \beta_{5} \\
& +\frac{1}{120} M t^{5} \gamma \beta_{2}^{2} \beta_{4} \beta_{5}+\frac{1}{120} N t^{5} \gamma \beta_{2}^{2} \beta_{4} \beta_{5} \\
& -\frac{1}{120} M t^{5} \gamma \beta_{1} \beta_{3} \beta_{4} \beta_{5}+\frac{1}{120} M t^{5} \gamma \beta_{2} \beta_{4}^{2} \beta_{5} \\
& +\frac{1}{120} M t^{5} \gamma^{2} \beta_{2} \beta_{5}^{2}+\frac{1}{120} M t^{5} \gamma \beta_{1} \beta_{2} \beta_{5}^{2} \\
& +\frac{1}{120} M t^{5} \gamma \beta_{2}^{2} \beta_{5}^{2}-\frac{1}{120} M t^{5} \gamma \beta_{1} \beta_{3} \beta_{5}^{2} \\
& +\frac{1}{120} M t^{5} \gamma \beta_{2} \beta_{4} \beta_{5}^{2}+\frac{1}{120} M t^{5} \gamma \beta_{2} \beta_{5}^{3} \text {, }
\end{aligned}
$$




$$
\begin{aligned}
& V_{S 5}[t]=\frac{1}{120} t^{5} \zeta \rho \beta_{1}^{2} \beta_{2} \beta_{4}-\frac{1}{120} N t^{5} \beta_{1}^{3} \beta_{2} \beta_{4} \\
& +\frac{1}{120} t^{5} \delta \lambda \beta_{4}^{3} \beta_{5}+\frac{1}{120} t^{5} \zeta \rho \beta_{4}^{3} \beta_{5} \\
& +\frac{1}{60} t^{5} \zeta \rho \beta_{1} \beta_{2}^{2} \beta_{4}-\frac{1}{40} N t^{5} \beta_{1}^{2} \beta_{2}^{2} \beta_{4} \\
& +\frac{1}{120} t^{5} \zeta \rho \beta_{2}^{3} \beta_{4}-\frac{1}{40} N t^{5} \beta_{1} \beta_{2}^{3} \beta_{4} \\
& -\frac{1}{120} N t^{5} \beta_{2}^{4} \beta_{4}-\frac{1}{120} t^{5} \zeta \rho \beta_{1}^{2} \beta_{3} \beta_{4} \\
& +\frac{1}{120} N t^{5} \beta_{1}^{3} \beta_{3} \beta_{4}-\frac{1}{120} t^{5} \zeta \rho \beta_{1} \beta_{2} \beta_{3} \beta_{4} \\
& +\frac{1}{60} N t^{5} \beta_{1}^{2} \beta_{2} \beta_{3} \beta_{4}+\frac{1}{120} N t^{5} \beta_{1} \beta_{2}^{2} \beta_{3} \beta_{4} \\
& -\frac{1}{120} t^{5} \zeta \rho \beta_{1} \beta_{3}^{2} \beta_{4}+\frac{1}{120} N t^{5} \beta_{1}^{2} \beta_{3}^{2} \beta_{4} \\
& +\frac{1}{120} N t^{5} \beta_{1} \beta_{2} \beta_{3}^{2} \beta_{4}+\frac{1}{120} t^{5} \delta \lambda \beta_{3}^{3} \beta_{4} \\
& +\frac{1}{120} N t^{5} \beta_{1} \beta_{3}^{3} \beta_{4}+\frac{1}{120} t^{5} \zeta \rho \beta_{1} \beta_{2} \beta_{4}^{2} \\
& -\frac{1}{120} N t^{5} \beta_{1}^{2} \beta_{2} \beta_{4}^{2}+\frac{1}{120} t^{5} \zeta \rho \beta_{2}^{2} \beta_{4}^{2} \\
& -\frac{1}{60} N t^{5} \beta_{1} \beta_{2}^{2} \beta_{4}^{2}-\frac{1}{120} N t^{5} \beta_{2}^{3} \beta_{4}^{2} \\
& -\frac{1}{120} t^{5} \zeta \rho \beta_{1} \beta_{3} \beta_{4}^{2}+\frac{1}{120} N t^{5} \beta_{1}^{2} \beta_{3} \beta_{4}^{2} \\
& +\frac{1}{120} N t^{5} \beta_{1} \beta_{2} \beta_{3} \beta_{4}^{2}+\frac{1}{120} t^{5} \delta \lambda \beta_{3}^{2} \beta_{4}^{2} \\
& +\frac{1}{120} N t^{5} \beta_{1} \beta_{3}^{2} \beta_{4}^{2}+\frac{1}{120} t^{5} \zeta \rho \beta_{2} \beta_{4}^{3} \\
& -\frac{1}{120} N t^{5} \beta_{1} \beta_{2} \beta_{4}^{3}-\frac{1}{120} N t^{5} \beta_{2}^{2} \beta_{4}^{3} \\
& +\frac{1}{120} t^{5} \delta \lambda \beta_{3} \beta_{4}^{3}+\frac{1}{120} N t^{5} \beta_{1} \beta_{3} \beta_{4}^{3} \\
& +\frac{1}{120} t^{5} \delta \lambda \beta_{4}^{4}+\frac{1}{120} t^{5} \zeta \rho \beta_{4}^{4}-\frac{1}{120} N t^{5} \beta_{2} \beta_{4}^{4} \\
& -\frac{1}{120} M t^{5} \gamma^{2} \beta_{2} \beta_{4} \beta_{5}-\frac{1}{120} M t^{5} \gamma \beta_{1} \beta_{2} \beta_{4} \beta_{5} \\
& +\frac{1}{120} t^{5} \zeta \rho \beta_{1} \beta_{2} \beta_{4} \beta_{5}-\frac{1}{120} N t^{5} \beta_{1}^{2} \beta_{2} \beta_{4} \beta_{5} \\
& -\frac{1}{120} M t^{5} \gamma \beta_{2}^{2} \beta_{4} \beta_{5}+\frac{1}{120} t^{5} \zeta \rho \beta_{2}^{2} \beta_{4} \beta_{5} \\
& -\frac{1}{60} N t^{5} \beta_{1} \beta_{2}^{2} \beta_{4} \beta_{5}-\frac{1}{120} N t^{5} \beta_{2}^{3} \beta_{4} \beta_{5} \\
& +\frac{1}{120} M t^{5} \gamma \beta_{1} \beta_{3} \beta_{4} \beta_{5}-\frac{1}{120} t^{5} \zeta \rho \beta_{1} \beta_{3} \beta_{4} \beta_{5} \\
& +\frac{1}{120} N t^{5} \beta_{1}^{2} \beta_{3} \beta_{4} \beta_{5}+\frac{1}{120} N t^{5} \beta_{1} \beta_{2} \beta_{3} \beta_{4} \beta_{5} \\
& +\frac{1}{120} t^{5} \delta \lambda \beta_{3}^{2} \beta_{4} \beta_{5}+\frac{1}{120} N t^{5} \beta_{1} \beta_{3}^{2} \beta_{4} \beta_{5} \\
& -\frac{1}{120} M t^{5} \gamma \beta_{2} \beta_{4}^{2} \beta_{5}+\frac{1}{120} t^{5} \zeta \rho \beta_{2} \beta_{4}^{2} \beta_{5} \\
& -\frac{1}{120} N t^{5} \beta_{1} \beta_{2} \beta_{4}^{2} \beta_{5}-\frac{1}{120} N t^{5} \beta_{2}^{2} \beta_{4}^{2} \beta_{5} \\
& +\frac{1}{120} t^{5} \delta \lambda \beta_{3} \beta_{4}^{2} \beta_{5}+\frac{1}{120} N t^{5} \beta_{1} \beta_{3} \beta_{4}^{2} \beta_{5} \\
& -\frac{1}{120} N t^{5} \beta_{2} \beta_{4}^{3} \beta_{5}-\frac{1}{60} M t^{5} \gamma \beta_{2} \beta_{4} \beta_{5}^{2} \\
& +\frac{1}{120} t^{5} \zeta \rho \beta_{2} \beta_{4} \beta_{5}^{2}-\frac{1}{120} N t^{5} \beta_{1} \beta_{2} \beta_{4} \beta_{5}^{2} \\
& -\frac{1}{120} N t^{5} \beta_{2}^{2} \beta_{4} \beta_{5}^{2}+\frac{1}{120} t^{5} \delta \lambda \beta_{3} \beta_{4} \beta_{5}^{2} \\
& +\frac{1}{120} N t^{5} \beta_{1} \beta_{3} \beta_{4} \beta_{5}^{2}+\frac{1}{120} t^{5} \delta \lambda \beta_{4}^{2} \beta_{5}^{2} \\
& +\frac{1}{120} t^{5} \zeta_{\rho} \beta_{4}^{2} \beta_{5}^{2}-\frac{1}{120} N t^{5} \beta_{2} \beta_{4}^{2} \beta_{5}^{2} \\
& +\frac{1}{120} t^{5} \delta \lambda \beta_{4} \beta_{5}^{3}+\frac{1}{120} t^{5} \zeta \rho \beta_{4} \beta_{5}^{3} \\
& -\frac{1}{120} N t^{5} \beta_{2} \beta_{4} \beta_{5}^{3}-\frac{1}{120} M t^{5} \beta_{5}^{5}, \\
& V_{I 5}[t]=\frac{1}{120} M t^{5} \gamma^{4} \beta_{5}-\frac{1}{120} t^{5} \gamma^{2} \delta \lambda \beta_{4} \beta_{5} \\
& -\frac{1}{120} t^{5} \gamma^{2} \zeta \rho \beta_{4} \beta_{5}+\frac{1}{120} N t^{5} \gamma^{2} \beta_{2} \beta_{4} \beta_{5} \\
& -\frac{1}{120} t^{5} \gamma \zeta \rho \beta_{2} \beta_{4} \beta_{5}+\frac{1}{120} N t^{5} \gamma \beta_{1} \beta_{2} \beta_{4} \beta_{5} \\
& -\frac{1}{120} t^{5} \zeta \rho \beta_{1} \beta_{2} \beta_{4} \beta_{5}+\frac{1}{120} N t^{5} \beta_{1}^{2} \beta_{2} \beta_{4} \beta_{5} \\
& +\frac{1}{120} N t^{5} \gamma \beta_{2}^{2} \beta_{4} \beta_{5}-\frac{1}{120} t^{5} \zeta \rho \beta_{2}^{2} \beta_{4} \beta_{5} \\
& +\frac{1}{60} N t^{5} \beta_{1} \beta_{2}^{2} \beta_{4} \beta_{5}+\frac{1}{120} N t^{5} \beta_{2}^{3} \beta_{4} \beta_{5} \\
& -\frac{1}{120} t^{5} \gamma \delta \lambda \beta_{3} \beta_{4} \beta_{5}-\frac{1}{120} N t^{5} \gamma \beta_{1} \beta_{3} \beta_{4} \beta_{5} \\
& +\frac{1}{120} t^{5} \zeta \rho \beta_{1} \beta_{3} \beta_{4} \beta_{5}-\frac{1}{120} N t^{5} \beta_{1}^{2} \beta_{3} \beta_{4} \beta_{5} \\
& -\frac{1}{120} N t^{5} \beta_{1} \beta_{2} \beta_{3} \beta_{4} \beta_{5}-\frac{1}{120} t^{5} \delta \lambda \beta_{3}^{2} \beta_{4} \beta_{5} \\
& -\frac{1}{120} N t^{5} \beta_{1} \beta_{3}^{2} \beta_{4} \beta_{5}-\frac{1}{120} t^{5} \gamma \delta \lambda \beta_{4}^{2} \beta_{5} \\
& -\frac{1}{120} t^{5} \gamma \zeta \rho \beta_{4}^{2} \beta_{5}+\frac{1}{120} N t^{5} \gamma \beta_{2} \beta_{4}^{2} \beta_{5} \\
& -\frac{1}{120} t^{5} \zeta \rho \beta_{2} \beta_{4}^{2} \beta_{5}+\frac{1}{120} N t^{5} \beta_{1} \beta_{2} \beta_{4}^{2} \beta_{5} \\
& +\frac{1}{120} N t^{5} \beta_{2}^{2} \beta_{4}^{2} \beta_{5}-\frac{1}{120} t^{5} \delta \lambda \beta_{3} \beta_{4}^{2} \beta_{5} \\
& -\frac{1}{120} N t^{5} \beta_{1} \beta_{3} \beta_{4}^{2} \beta_{5}-\frac{1}{120} t^{5} \delta \lambda \beta_{4}^{3} \beta_{5} \\
& -\frac{1}{120} t^{5} \zeta \rho \beta_{4}^{3} \beta_{5}+\frac{1}{120} N t^{5} \beta_{2} \beta_{4}^{3} \beta_{5} \\
& +\frac{1}{120} M t^{5} \gamma^{3} \beta_{5}^{2}-\frac{1}{120} t^{5} \gamma \delta \lambda \beta_{4} \beta_{5}^{2} \\
& -\frac{1}{120} t^{5} \gamma \zeta \rho \beta_{4} \beta_{5}^{2}+\frac{1}{120} M t^{5} \gamma \beta_{2} \beta_{4} \beta_{5}^{2} \\
& +\frac{1}{120} N t^{5} \gamma \beta_{2} \beta_{4} \beta_{5}^{2}-\frac{1}{120} t^{5} \zeta \rho \beta_{2} \beta_{4} \beta_{5}^{2} \\
& +\frac{1}{120} N t^{5} \beta_{1} \beta_{2} \beta_{4} \beta_{5}^{2}+\frac{1}{120} N t^{5} \beta_{2}^{2} \beta_{4} \beta_{5}^{2}
\end{aligned}
$$




$$
\begin{aligned}
& -\frac{1}{120} t^{5} \delta \lambda \beta_{3} \beta_{4} \beta_{5}^{2}-\frac{1}{120} N t^{5} \beta_{1} \beta_{3} \beta_{4} \beta_{5}^{2} \\
& -\frac{1}{120} t^{5} \delta \lambda \beta_{4}^{2} \beta_{5}^{2}-\frac{1}{120} t^{5} \zeta \rho \beta_{4}^{2} \beta_{5}^{2} \\
& +\frac{1}{120} N t^{5} \beta_{2} \beta_{4}^{2} \beta_{5}^{2}+\frac{1}{120} M t^{5} \gamma^{2} \beta_{5}^{3} \\
& -\frac{1}{120} t^{5} \delta \lambda \beta_{4} \beta_{5}^{3}-\frac{1}{120} t^{5} \zeta \rho \beta_{4} \beta_{5}^{3} \\
& +\frac{1}{120} N t^{5} \beta_{2} \beta_{4} \beta_{5}^{3}+\frac{1}{120} M t^{5} \gamma \beta_{5}^{4} \\
& +\frac{1}{120} M t^{5} \beta_{5}^{5} .
\end{aligned}
$$

In general, by the repeated application of the above procedure, we have that

$$
\begin{gathered}
H_{S n}=\frac{t^{n}}{n !} a_{1 n}, \quad H_{E n}=\frac{t^{n}}{n !} a_{2 n}, \quad H_{I n}=\frac{t^{n}}{n !} a_{3 n}, \\
V_{S n}=\frac{t^{n}}{n !} a_{4 n}, \quad V_{I n}=\frac{t^{n}}{n !} a_{5 n},
\end{gathered}
$$

where $a_{1 n}, a_{2 n}, a_{3 n}, a_{4 n}, a_{5 n}$ depend on fixed set of empirical parameters. Hence, the approximate solution of the model above is given as

$$
\begin{aligned}
& H_{S}(t)=\sum_{n=0}^{\infty} \frac{t^{n}}{n !} a_{1 n}, \\
& H_{E}(t)=\sum_{n=0}^{\infty} \frac{t^{n}}{n !} a_{2 n}, \\
& H_{I}(t)=\sum_{n=0}^{\infty} \frac{t^{n}}{n !} a_{3 n}, \\
& V_{S}(t)=\sum_{n=0}^{\infty} \frac{t^{n}}{n !} a_{4 n}, \\
& V_{I}(t)=\sum_{n=0}^{\infty} \frac{t^{n}}{n !} a_{5 n} .
\end{aligned}
$$

We therefore use the following theoretical parameters $\beta_{1}=$ $0.5, \beta_{2}=0.5, \beta_{3}=0.5, \beta_{4}=0.5, \beta_{5}=0.5, \gamma=1, \zeta=1$, $N=10, M=10, \rho=1, \delta=1$, and $\lambda=1$ to examine the behavior of the five different compartmental numerical solutions in the system. Figure 3 shows the behavior of the numerical solution.

\section{Conclusion}

Onchocerciasis model was examined and the mathematical formulation underpinning the spread and control of this disease has been proposed on one hand. On the other hand, we made use of some new analytical methods called the homotopy decomposition method (HDM) to derive the solution of the resulting set of (1) to (5). The numerical results were presented to test the efficiency and the accuracy of the method used. The mathematical analysis of the resulting set of (1) to (5) above revealed that the stability analysis of the

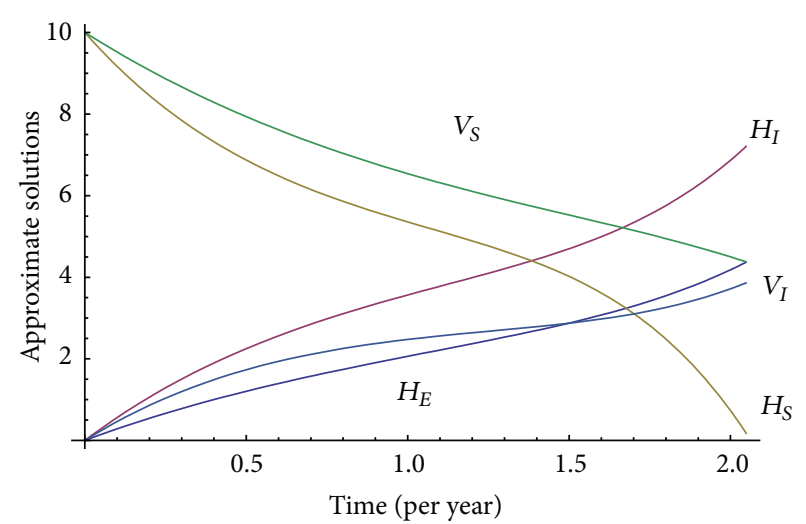

Figure 3: Numerical solution of the model.

five roots of the characteristics equations has all negative real part. In particular, given that the stability analysis shows that the DFE is asymptotically stable, it would be possible that the parasite Onchocerca volvulus be eradicated in a finite time. On the other hand, the numerical solution confirmed that as the number of humans and vectors in the susceptible compartments reduces, the number of infective humans and vectors in the infectious compartment increases. The numerical solutions in both cases displayed the biological behaviour of the real world situation. Therefore, in order to build a healthy and a sustainable population, treatment with Ivermectin/Mectizan drugs and a more hygienic environment that does not allow the breeding and survival of blackflies could lead to a total eradication of the disease.

\section{Conflict of Interests}

The authors declare that they have no conflict of interests regarding the publication of this paper.

\section{References}

[1] J. H. F. Remme, The Global Burden of Onchocerciasis in 1990, World Health Organization, Geneva, Switzerland, 2004, http:// www.who.int/healthinfo/global_burden_disease/Onchocerciasis\%201990.pdf.

[2] World Health Organization, "Prevention of blindness and visual impairment: priority eye diseases," 2014, http://www.who.int/ blindness/causes/priority/en/index3.html.

[3] World Health Organization, Certification of Elimination of Human Onchocerciasis: Criteria and Procedures, World Health Organization, Geneva, Switzerland, 2001, http://whqlibdoc.who .int/hq/2001/WHO_CDS_CPE_CEE_2001.18b.pdf.

[4] K. A. Lindblade, B. Arana, G. Zea-Flores et al., "Elimination of Onchocerca volvulus transmission in the Santa Rosa focus of Guatemala," The American Journal of Tropical Medicine and Hygiene, vol. 77, pp. 334-341, 2007.

[5] J. C. Anosike and C. O. E. Onwuliri, "Studies on filariasis in Bauchi State, Nigeria: 1. Endemicity of human onchocerciasis in Ningi Local Government Area," Annals of Tropical Medicine and Parasitology, vol. 89, no. 1, pp. 31-38, 1995.

[6] World Health Organization, Weekly Epidemiological Records, 71, WHO, Geneva, Switzerland, 1993. 
[7] World Health Organization, http://www.who.int/neglected_diseases/en/.

[8] Sightsaver, http://www.sightsavers.org/about us/publications/ onchocerciasis.

[9] E. C. Uttah, "Onchocerciasis in the Upper Ima River Basin," Iranian Journal of Parasitology, vol. 5, no. 2, pp. 33-41, 2010.

[10] J. C. Anosike and C. O. E. Onwuliri, "Studies on filariasis in Bauchi State, Nigeria-1: endemicity of human onchocerciasis in Ningi Local Government Area," Annals of Tropical Medicine and Parasitology, vol. 89, no. 1, pp. 31-38, 1995.

[11] L. D. Edungbola and S. O. Asaolu, "Parasitologic survey of onchocerciasis (river blindness) in Babana district, Kwara State, Nigeria," American Journal of Tropical Medicine and Hygiene, vol. 33, no. 6, pp. 1147-1154, 1984.

[12] O. C. Nwaorgu, A. Ohaegbula, I. E. Onweluzo et al., "Results of a large scale onchocercosis survey in Enugu State, Nigeria," Journal of Helminthology, vol. 68, no. 2, pp. 155-159, 1994.

[13] F. O. Akinbo and C. E. Okaka, "Prevalence and socio-economic effects of onchocerciasis in Ovia North East Local Government Area of Edo State, Nigeria," Rivista Di Parasitologie, vol. 22, no. 3, pp. 215-221, 2005.

[14] M. D. Wogu and C. E. Okaka, "Prevalence and socioeconomic effects of onchocerciasis in Okpuje, Owan West Local Government Area, Edo State, Nigeria," International Journal of Biomedical and Health Sciences, vol. 4, no. 3, 2008, http://www.asopah.org/.

[15] S. C. O. Noutchie, "Homotopy Decomposition analysis of a tuberculosis model," Life Science Journal, vol. 10, no. 3, pp. 459465, 2013.

[16] A. Atangana and A. Secer, "The time-fractional coupledKorteweg-de-Vries equations," Abstract and Applied Analysis, vol. 2013, Article ID 947986, 8 pages, 2013.

[17] A. Atangana and N. Bildik, "Approximate solution of tuberculosis disease population dynamics model," Abstract and Applied Analysis, vol. 2013, Article ID 759801, 8 pages, 2013. 


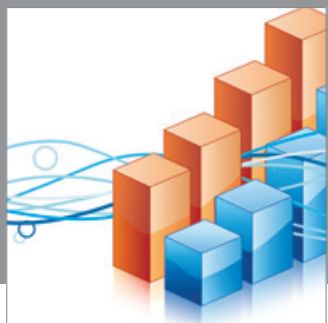

Advances in

Operations Research

mansans

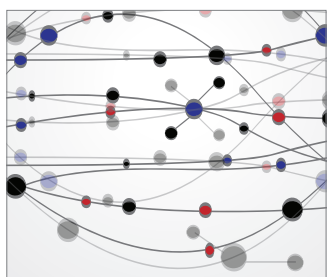

The Scientific World Journal
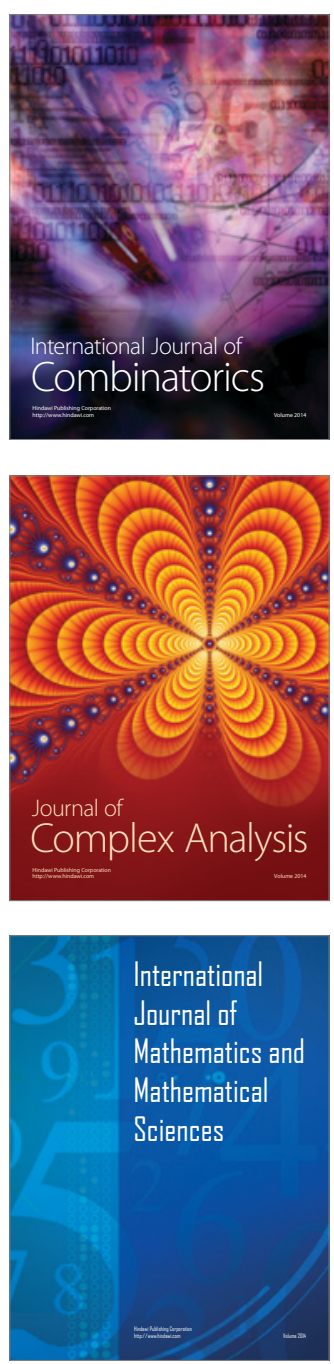
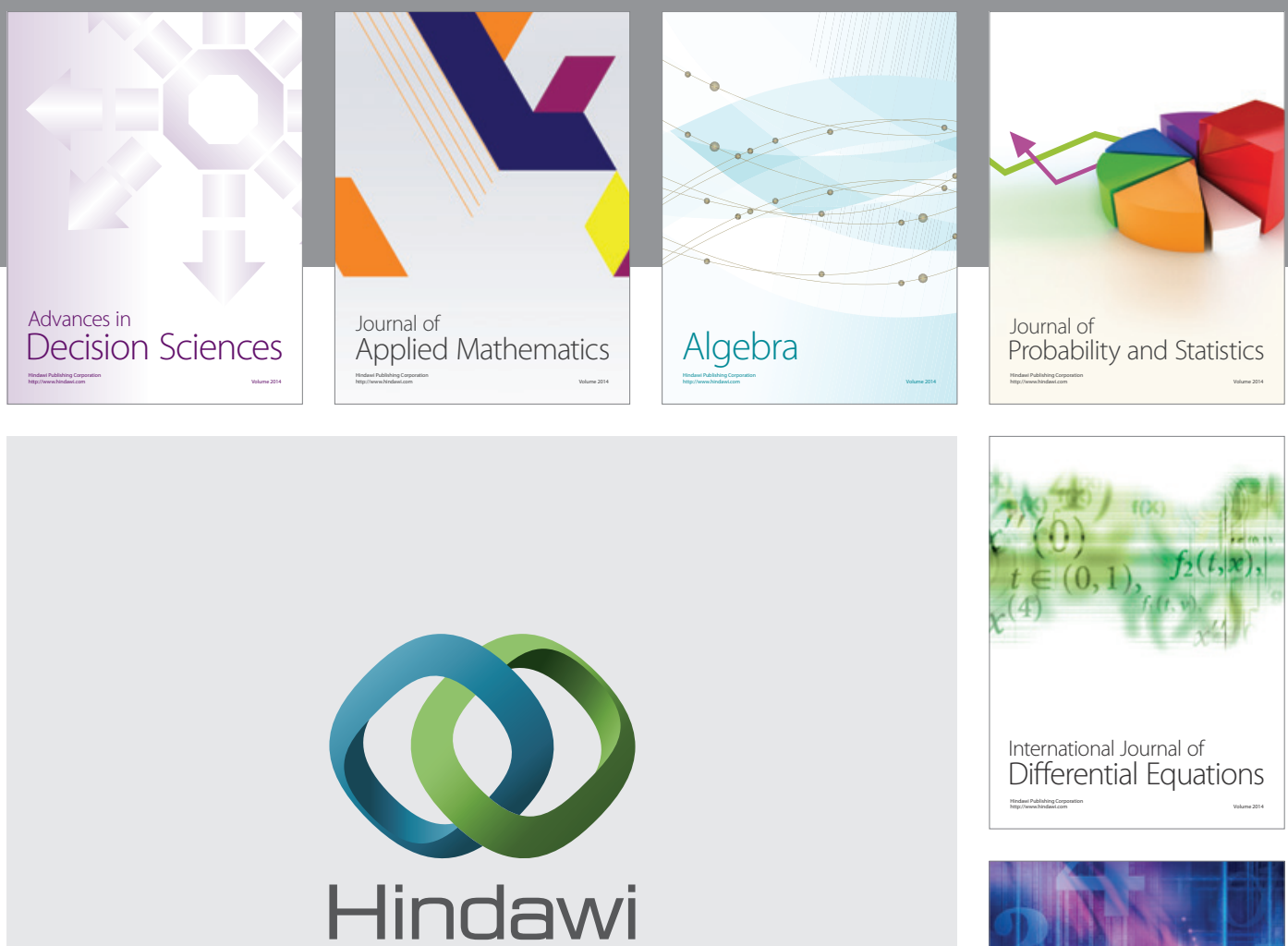

Submit your manuscripts at http://www.hindawi.com
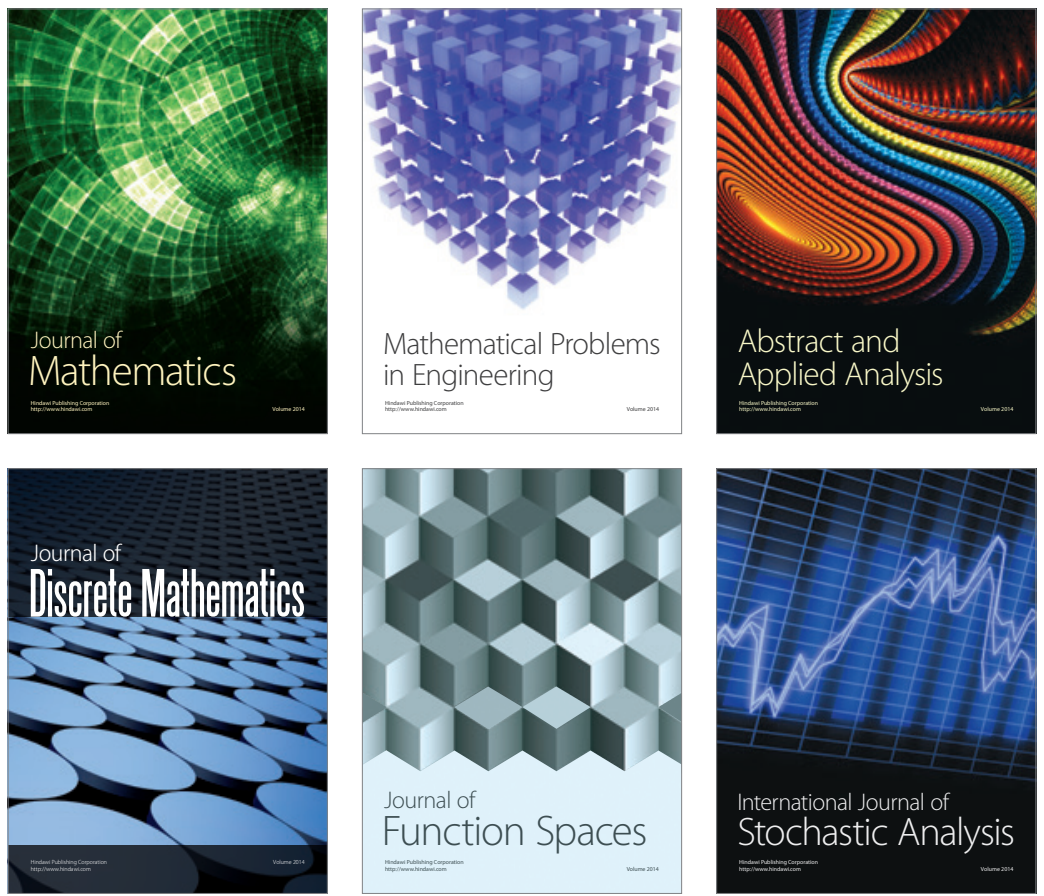

Journal of

Function Spaces

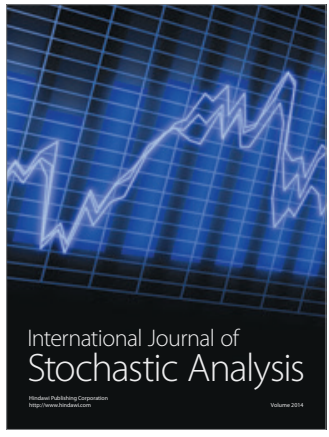

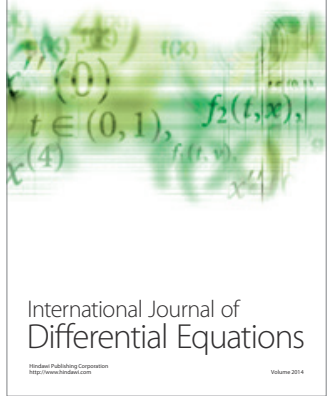
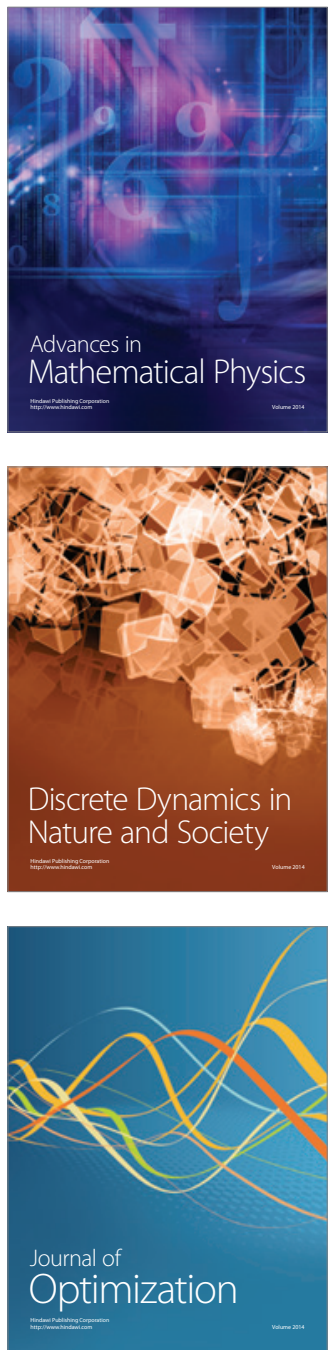